\title{
Interstellar cloud structure: the statistics of centroid velocities
}

\author{
V. Ossenkopf ${ }^{1,2}$, A. Esquivel $^{3}$, A. Lazarian $^{3}$, and J. Stutzki ${ }^{1}$ \\ 1 I. Physikalisches Institut der Universität zu Köln, Zülpicher Straße 77, 50937 Köln, Germany \\ e-mail: ossk@ph1.uni-koeln.de \\ 2 SRON National Institute for Space Research, PO Box 800, 9700 AV Groningen, The Netherlands \\ 3 Astronomy Department, University of Wisconsin-Madison, 475 N. Charter St., Madison, WI 53706, USA
}

Received 24 January 2005 / Accepted 20 January 2006

\section{ABSTRACT}

\begin{abstract}
Context. The statistical properties of maps of line centroids have been used for almost 50 years, but there is still no general agreement on their interpretation.

Aims. We have tried to quantify which properties of underlying turbulent velocity fields can be derived from centroid velocity maps, and we tested conditions under which the scaling behaviour of the centroid velocities matches the scaling of the three-dimensional velocity field.

Methods. Using fractal cloud models we systematically studied the relation between three-dimensional density and velocity fields and the statistical properties of the resulting line centroid maps. We paid special attention to cases with large density fluctuations resembling supersonic interstellar turbulence. Starting from the $\Delta$-variance analysis, we derived a new tool to compute the scaling behaviour of the three-dimensional velocity field from observed intensity and centroid velocity maps.

Results. We provide two criteria to decide whether the information from the centroid velocities directly reflects the properties of the underlying velocity field. Applying these criteria allows us to understand the different results found so far in the literature for interpreting the statistics of velocity centroids. The new iteration scheme can be used to derive the three-dimensional velocity scaling from centroid velocity maps for arbitrary density and velocity fields, but it requires accurate knowledge of the average density of the interstellar cloud under consideration.
\end{abstract}

Key words. ISM: clouds - ISM: kinematics and dynamics - ISM: structure - methods: statistical

\section{Introduction}

Understanding the role and nature of interstellar turbulence has been the subject of intensive studies for half a century now, but many aspects still remain open (cf. Elmegreen \& Scalo 2004). Major questions concern the mechanisms by which turbulent motions are driven and the role of the strong compressibility of the interstellar medium for the structure of the turbulent energy cascade. Both aspects are directly reflected on the spectrum of velocity fluctuations in the turbulent motion. It is frequently claimed that driving mechanisms should create dominant motions at the corresponding scales, and the power spectrum of velocities in the turbulent cascade is known to change from a $P(|\boldsymbol{k}|) \propto|\boldsymbol{k}|^{-11 / 3}$ Kolmogorov spectrum for an incompressible medium to a $P(|\boldsymbol{k}|) \propto|\boldsymbol{k}|^{-4}$ spectrum of Burger's turbulence in a highly compressible medium dominated by shocks (Chappell \& Scalo 1999). However, numerical simulations often show a different behaviour (see Cho \& Lazarian 2005), which makes the issue of the observed spectrum very intriguing.

To support the theoretical understanding of the interstellar turbulence, it is thus essential to actually measure the velocity structure in the interstellar medium. Unfortunately, there is no direct way to do this. Observations of the profiles of atomic or molecular lines from interstellar clouds allow information on the line-of-sight velocity structure of the clouds to be deduced. The problem of recovering the velocity information from lines is far from being straightforward. Even in the simplest case of thermally-excited, optically-thin lines from an isothermal medium the line profiles originate from a convolution of the density structure $\rho$ depending on the sky coordinates $\boldsymbol{x}=(\alpha, \delta)$ and the line-of-sight coordinate $z$ with the velocity structure $v_{z}(\boldsymbol{x}, z)$ :

$I(\boldsymbol{x}, v) \propto \int \mathrm{d} z \rho(\boldsymbol{x}, z) \phi\left(v-v_{z}(\boldsymbol{x}, z)\right)$.

In the limit of narrow lines, the line profile $\phi\left(v-v_{z}(\boldsymbol{x}, z)\right)$ can be approximated by a $\delta$-function. There are several complementary ways to use this information (cf. Lazarian 2004). Here, we restrict ourselves to centroids, the first moment of the lines, but the centroids still provide no direct map of the velocity structure.

Models for the density structure and the relation between density and velocity structure are needed to deduce the latter from the profiles $I(\boldsymbol{x}, v)$. This is straightforward for simple geometries like spherical clouds or thin disks but extremely difficult for filamentary, turbulent cloud structures showing varying substructures on all spatial scales. Hydrodynamic or magnetohydrodynamic numerical simulations can be used as physically justified models for turbulent interstellar clouds within a limited dynamic range. The nature of these can be described, however, only in terms of statistical measures. Fractal cloud models provide a reasonable phenomenological description of the clouds. We focus on measures for the spatial scaling of the velocity structure. The ultimate goal is to derive the three-dimensional (3D) power spectrum of velocity fluctuations.

A recovery of $3 \mathrm{D}$ information from the available $2 \mathrm{D}$ data requires, in general, an inversion, which may result in substantial noise in the inverted data. To derive the turbulence statistics we can, however, use its symmetries. Here, we restrict ourselves to statistically isotropic turbulence. The derivation of 
properties of anisotropic, but axisymmetric turbulence from observations was discussed by Lazarian (1995). Anisotropies can be due to the magnetic fields (Higdon 1984; Zank \& Matthaeus 1992; Goldreich \& Shridhar 1995). However, if, as both theory and numerics suggest (see Goldreich \& Shridhar 1995; Cho \& Lazarian 2003), the energy spectrum is dominated by fluctuations perpendicular to the local direction of magnetic field, the effects of anisotropy on the observed spectra can be neglected (Esquivel et al. 2003).

In order to derive the isotropic power spectrum, we use an auxiliary quantity, the $\Delta$-variance spectrum, because of its practical advantages when measuring the velocity scaling in observed data. Moreover, we restrict the analysis here to the first moments of the lines, the centroid velocity, as the most obvious tracer for measuring the velocity structure in an interstellar cloud.

Maps of observed line centroids have been systematically studied to obtain the scaling behaviour of centroid velocity differences as a function of lag for almost 50 years now (e.g. Münch 1958; Kleiner \& Dickman 1985; Miesch \& Bally 1994; Lis et al. 1996; Miesch et al. 1999). However, there is still no agreement on the theoretical relation between the observed scaling behaviour of the centroid velocities and the scaling behaviour of the underlying turbulent velocity structure. Although it was clear from the very beginning that density structure can influence the line centroids, until the recent past there was no criterion for estimating the quantitative effect of the density.

When investigating hydrodynamic turbulence simulations, Ossenkopf \& Mac Low (2002) found that the centroid maps show approximately the same Hurst index, i.e. the same relative variation across a given scale, as the underlying $3 \mathrm{D}$ velocity structure. This means that the power spectral index in their centroid maps was reduced by one compared to the power spectral index in the 3D velocity structure. Studies of fractal clouds by Miville-Deschênes et al. (2003a) showed in contrast that their centroid maps have the same power spectral index as the $3 \mathrm{D}$ velocity structure ${ }^{1}$. Lazarian \& Esquivel (2003) provided an analytical treatment of the centroid statistics introducing a new, more robust definition of velocity centroids, and formulated a criterion where the centroids represent the velocity statistics, but this publication did not cover the parameter space so as to be fairly compared with previous studies. The problem was further elaborated in a subsequent study by Levrier (2004), who pointed out that the statistical treatment presented in the form of structure functions by Lazarian \& Esquivel (2003) may have some advantages if rewritten in terms of correlation functions. By assuming that the fluctuations are small compared to the mean density, he obtained analytic expressions for the correlation functions of centroids. In combining structure and correlation functions Esquivel \& Lazarian (2005) provided a detailed study of centroid velocities for data obtained through compressible MHD simulations. Here, we compare the different centroid definitions and test their outcome for a set of fractal cloud models.

Using the $\Delta$-variance analysis, we show that it is applicable to deriving the velocity power spectrum from observed centroid maps but that the reliability of this derivation depends critically on individual turbulence parameters. The centroid maps reflect the actual velocity distribution only in a medium with an average

1 When dealing with projected quantities, one has to carefully distinguish correlation functions and power spectra. When a power-law approximation is good for both of them, the spectral index of correlation functions gets steeper by one due to projection, while the 2D projected power spectrum retains the spectral index of the underlying 3D spectrum. density that is large compared to the density dispersion. Here, the $\Delta$-variance analysis provides a direct measure for the power spectral index of the velocity structure. Only when applied in an iterative process with an a priori knowledge of the average density, the analysis of centroid maps allows one to approximate the velocity structure in the general case. The steeper the velocity spectrum and the better we know the average density, the better the approximation.

In Sect. 2 we briefly repeat the formalism used to describe the velocity centroids, and discuss the properties of the test data sets and the ways to measure their spatial scaling behaviour in terms of the $\Delta$-variance. In Sect. 3 we analyse the centroid maps using the $\Delta$-variance, compare the results with the original test data, and derive criteria when the centroid maps can be used to directly measure the three-dimensional velocity structure. In Sect. 4 we propose an iterative method to derive the power spectrum of the velocity structure from the centroid maps in cases without a direct matching. Section 5 summarises our conclusions on the interpretation of observed data.

\section{The starting point}

\subsection{Definition of centroid velocities}

For the fluctuating density and velocity fields in a cloud, we can always write

$\rho(\boldsymbol{x}, z)=\rho_{0}+\delta \rho$

$v(\boldsymbol{x}, z)=v_{0}+\delta v$

where $\rho_{0}$ and $v_{0}$ are averages over the whole cloud, and $\delta \rho$ and $\delta v$ denote the variations across the cloud ${ }^{2}$.

When we assume that the emissivity is proportional to the density of the cloud, the line intensity $I(\boldsymbol{x}, v)$ at velocity $v$ is a measure for the total column density of emitters with this velocity at a given line-of-sight $\boldsymbol{x}$. This condition is violated for optically thick lines or media with strongly varying temperatures but it is fulfilled well e.g. for the [CII] emission from the cold neutral medium or the HI emission from the warm neutral medium. The effect of self-absorption will be quantified in a subsequent paper. For constant emissivity, the integrated line intensity is

$$
\begin{aligned}
I_{\text {int }}(\boldsymbol{x}) & =\int \mathrm{d} v I(\boldsymbol{x}, v) \\
& =X \int \mathrm{d} z \rho(\boldsymbol{x}, z)
\end{aligned}
$$

where $X$ is the proportionality factor from Eq. (1) translating the column density into a line intensity.

There are two different centroid definitions in common use. Ordinary centroid velocities, also known as normalised centroids, are obtained as

$$
\begin{aligned}
v_{\mathrm{c}, \text { norm }}(\boldsymbol{x}) & =\int \mathrm{d} v v I(\boldsymbol{x}, v) \mid \int \mathrm{d} v I(\boldsymbol{x}, v) \\
& =\int \mathrm{d} z\left(v_{0}+\delta v\right)\left(\rho_{0}+\delta \rho\right) / \int \mathrm{d} z\left(\rho_{0}+\delta \rho\right) .
\end{aligned}
$$

Unfortunately, this definition implies a complex combination of density and velocity fluctuations, which makes it impossible to

\footnotetext{
2 From here on we drop the index $z$ in the notation for the lineof-sight component of the velocity because we consider only this component.
} 
disentangle the influence from both structures in the general case. Only in case of very small fluctuations can a linearisation technique be developed (Levrier 2004). A better separation of density and velocity fluctuations in the centroids is obtained when we apply the definition of weighted, i.e. unnormalised, centroids as proposed by Lazarian \& Esquivel $(2003)^{3}$

$$
\begin{aligned}
v_{\mathrm{c}}(\boldsymbol{x})= & 1 / X \int \mathrm{d} v v I(\boldsymbol{x}, v) \\
= & v_{0} \rho_{0} z_{\mathrm{tot}}+\rho_{0} \int \mathrm{d} z \delta v \\
& +v_{0} \int \mathrm{d} z \delta \rho+\int \mathrm{d} z \delta \rho \delta v
\end{aligned}
$$

where $z_{\text {tot }}$ is the total thickness of the cloud. In this definition the centroids do not have the dimension of a velocity but of velocity times column density. For a better comparison with the ordinary centroid velocities, it is useful to normalise the weighted centroids by the average column density $\rho_{0} z_{\text {tot }}$, but we omit this factor in the following to keep the equations shorter. The constant factor would not change any of our conclusions on the scaling behaviour of the velocity structure.

We see that even in this definition the centroid velocities are not simply determined by the projected velocities $v_{0}+\int \mathrm{d} z \delta v$ but also by two terms reflecting the density variations. The contribution from the projected density variations $\int \mathrm{d} z \delta \rho$ can be easily obtained from the integrated line profiles, and it can be eliminated by selecting a velocity scale with $v_{0}=0$. However, the term containing the product of the fluctuations in the density and the velocity structure cannot be measured separately.

The scaling behaviour of the centroid velocities depends on the combination of density and velocity variations along the line of sight, which cannot be retrieved directly. The relative contribution of the simple projection of the velocity structure and the density variations $\delta \rho$ across the line of sight depends on the ratio between the density fluctuations $\delta \rho$ and the average density $\rho_{0}$. Equation (5) thus shows already that the ratio between the density dispersion $\sigma_{\rho}$ and the average density $\rho_{0}$ is a critical parameter for the relation between the $3 \mathrm{D}$ velocity scaling and the centroid scaling.

\subsection{Test data sets}

To study the general ability of different methods to extract the underlying velocity structure from observed centroid velocities, we constructed well defined test data sets for the density and velocity structure that were used to study the translation of their scaling properties into centroid properties.

Interstellar cloud observations often reveal self-similar scaling properties (e.g. Falgarone et al. 1995; Combes 2000) corresponding to power-law power spectra of the intensity distribution. Such intensity maps can be approximately modelled by fractional Brownian motion (fBm) structures (see e.g. Stutzki et al. 1998; Bensch et al. 2001). They are defined by the single number $\beta$ determining the exponent of the power spectrum, $P(|\boldsymbol{k}|) \propto|\boldsymbol{k}|^{-\beta}$. The phases of the Fourier spectrum are random.

Thus fBm's represent one of the simplest possible representations of interstellar cloud structures still allowing a parameter

\footnotetext{
3 In contrast to the original definition, we have not included the constant factor $X$ in the centroid definition so that the weighted centroids have the dimension of a velocity-times-column-density here instead of velocity-times-intensity. This keeps the equations in the following sections somewhat shorter.
}

study in terms of the spectral index $\beta$ that determines the actual appearance of the structures. The fBm's can be defined in arbitrary dimensions and we used their essential property that the projection of an $\mathrm{fBm}$ to lower dimensions results in a new $\mathrm{fBm}$ with the same spectral index (Stutzki et al. 1998; Brunt \& Mac Low 2004) ${ }^{4}$. Thus the spectral index measured for the column density directly reflects the index of the three-dimensional density structure.

Measured spectral indices for the column density structure of interstellar clouds range from 2.0 to 3.7 (Elmegreen $\&$ Scalo 2004; Falgarone et al. 2004). Observations of large molecular clouds and molecular cloud complexes and HI absorption line studies have provided typical values between 2.4 and 2.9 (e.g. Stenholm 1984; Langer et al. 1993; Deshpande et al. 2000; Bensch et al. 2001; Huber 2002; Padoan et al. 2003), whereas Bensch et al. (2001) found indications of somewhat larger indices at the scales of cloud cores. Observations of the warm atomic gas provided typical values between 3.3 (e.g. Stanimirović \& Lazarian 2001) and 3.6 (Miville-Deschênes et al. 2003b) with some indications of an even broader range from $8 / 3$ to $11 / 3$ in the LMC (Elmegreen et al. 2001). Due to a lack of direct measurements, as discussed in the introduction, the index range of the velocity structure is still hardly known. MHD simulations by Cho \& Lazarian (2003) indicate that it should be close to the Kolmogorov value of 11/3. In contrast, Ossenkopf \& Mac Low (2002) and Brunt \& Heyer (2002) obtained velocity spectral indices close to four from observations of the Polaris Flare molecular cloud and of molecular clouds in the FCRAO survey of the Outer Galaxy, respectively, consistent with the properties of a shock-dominated medium. In these cases, the velocity spectrum was always steeper than the column density spectrum. Here, we do not aim at reproducing the exact combination of spectral indices for any particular interstellar cloud, but want to study the general behaviour covering the full range of spectral indices observed so far.

Esquivel \& Lazarian (2005) demonstrated that the centroid structure function shows a qualitatively different behaviour for spectra with an index above and below 3.0 (steep and shallow spectra). Unfortunately, the observational data do not rule out either of the two types. Thus we focus on two test data sets: fBm's with a spectral index of 3.7 representing steep spectra and with an index of 2.6 representing a shallow behaviour. They sample both regimes and are close to some observed values for the velocity and density structure. We have studied a much larger parameter range covering spectral indices between 2.0 and 4.0; but with the four possible mutual combinations of the two spectral indices mentioned, all major effects are covered so that we restrict ourselves to these cases for all examples given in the following.

In Fig. 1 we give a visual impression of the difference in the actual projected structure between fBm's of a different spectral index. The spectral index basically determines the relative contribution of structures on different size scales. The fBm with an index of 2.6 shows a large amount of small-scale clumps and filaments, whereas the $\mathrm{fBm}$ with $\beta=3.7$ basically consists of one peak with fragmented boundaries.

The figure also reveals a general problem of fBm's when interpreted as density structure. They show negative values. On average fBm's have a Gaussian probability distribution with vanishing mean so that negative values can only be avoided when

\footnotetext{
4 As discussed by Stutzki et al. (1998) it is easy to show that this $\mathrm{fBm}$ property violates the often used hypothesis that the fractal dimension decreases by one in projection (Peitgen \& Saupe 1988).
} 

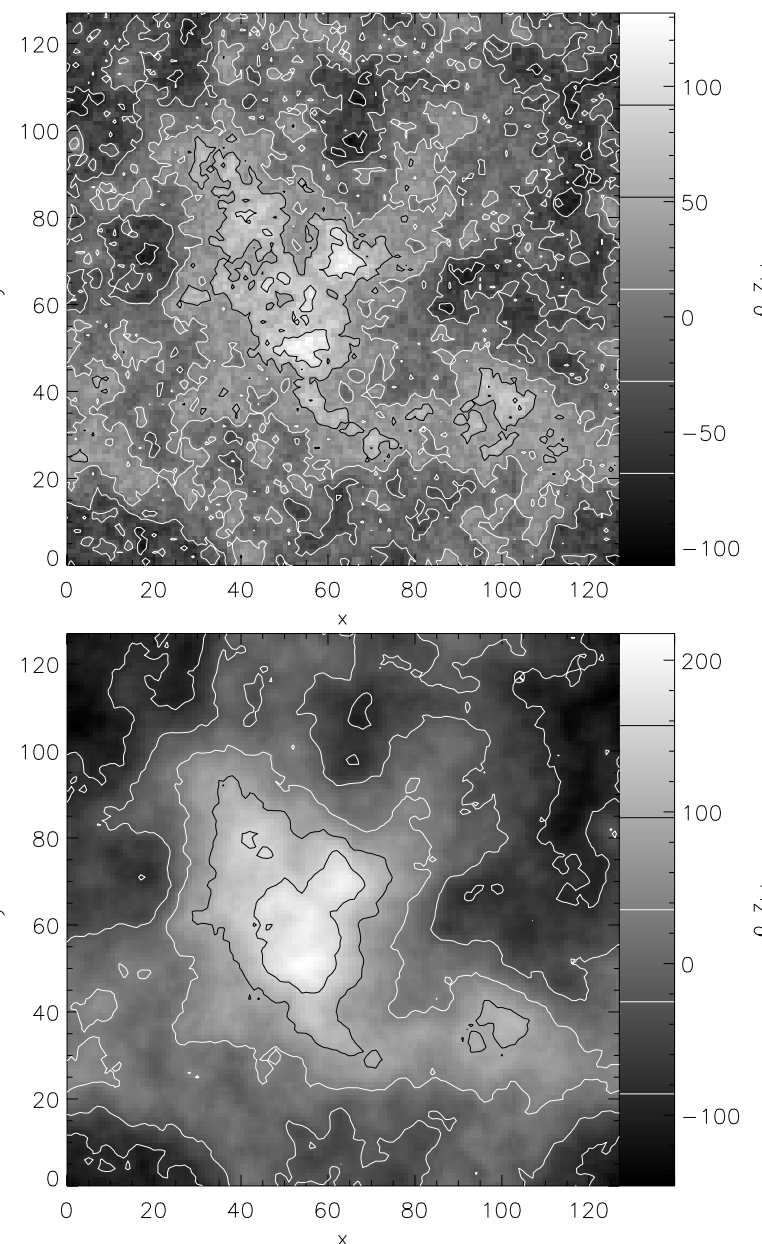

Fig. 1. Projected maps of $\mathrm{fBm}$ structures with spectral indices $\beta=2.6$ (upper plot) and $\beta=3.7$ (lower plot). Both data sets use the same random phases leading to the apparent similarity of the overall distribution in this example.

adding a large constant density offset. However, we drastically change the ratio $\sigma_{\rho} / \rho_{0}$ for the data set in this way. Another method to create a density distribution containing only positive values is to square or exponentiate the original $\mathrm{fBm}$ as proposed by Stutzki et al. (1998). Miville-Deschênes et al. (2003a) have claimed that exponentiation, $\rho_{\exp }=\rho_{0} \exp \left(a \rho_{\mathrm{fBm}}\right)$, does not affect the power-spectrum, but it is mathematically obvious that it can potentially destroy the power-law scaling. Thus we have tested the impact of exponentiation for different spectral indices $\beta$ and different factors $a$ translating the standard deviation of the $\mathrm{fBm}$ into the logarithmic standard deviation of the new density structure. The result is shown in Fig. 2 for an $\mathrm{fBm}$ with $\beta=4$ as used in Fig. 11 of Miville-Deschênes et al. (2003a) and for an $\mathrm{fBm}$ with $\beta=2.6$ in terms of $\Delta$-variance spectra (see Sect. 2.3).

It is obvious, that for narrow distributions, the distortion of the original spectrum by exponentiation is small, as the exponentiation is then close to a linear transformation. In general, we have to acknowledge, however, considerable distortions of the spectrum by the exponentiation. When creating a very wide density distribution from the $\beta=2.6 \mathrm{fBm}$, we even find a completely different scaling behaviour that instead resembles a structure with $\beta=0$. The example from Miville-Deschênes et al. (2003a) corresponds approximately to the $\beta=4, \sigma_{\log }=0.3$ case shown in Fig. 2. Here, the deviation from the original spectrum
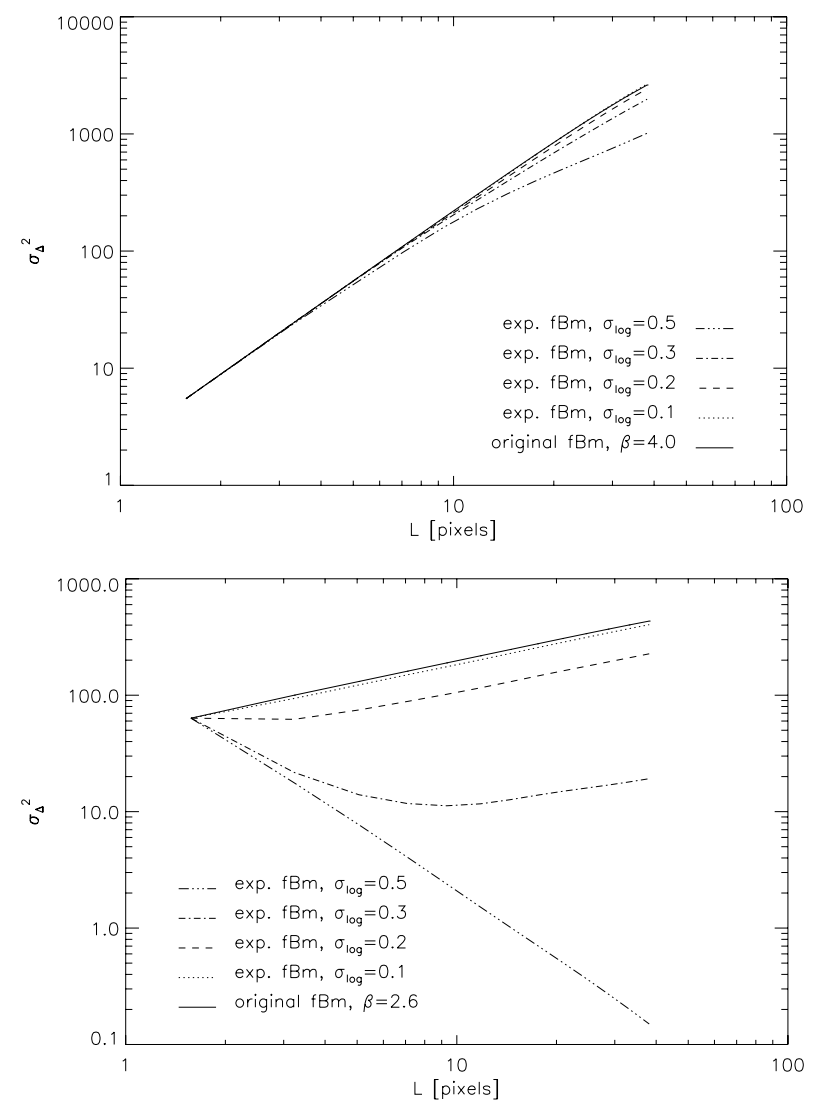

Fig. 2. $\Delta$-variance spectra of the projected structure of exponentiated $\mathrm{fBm}$ 's compared to the spectrum of the original $\mathrm{fBm}$. The upper plot represents $\beta=4$, the lower plot $\beta=2.6$. The different lines indicate different stretching factors $a$ resulting in different logarithmic widths of the distributions. The average logarithmic density is taken to be 2.0 in all cases.

is small so that it was not detectable. Moreover, we have found that the $\Delta$-variance reacts much more sensitively to the exponentiation than does the azimuthally averaged power spectrum. Only for very wide distributions and low spectral indices does the azimuthally averaged power spectra show similar noticeable deviations. In general we have to conclude that exponentiation leads to a change of the scaling properties. Consequently, nonlinear transformations are not well suited to produce well defined test data for the density structure. We will stick to the simple approach of adding a constant to the $\mathrm{fBm}$ and ignoring the remaining negative values for the construction of the density structure. The implications for this approach are quantified in detail in Sect. 3.1. In contrast to the density structure that has to be positively defined and necessarily has a non zero mean $\rho_{0}$, the velocity structure can use fBm's directly thus guaranteeing a zero value for $v_{0}$ so that the simplifications discussed above apply.

When using independent fBm's to represent both the density and the velocity structure of interstellar clouds, however, we neglect the interrelation of both quantities in the interstellar medium determined by the hydrodynamic equations, especially by the Poisson equation. Comparisons with magnetohydrodynamic simulations by Esquivel \& Lazarian (2005) have shown that the cross-correlation between the density and velocity fields has a negligible effect on the centroid velocities so that we can neglect its impact here. We will further discuss the influence of cross-correlations between density and velocity structure on different observational parameters in a subsequent paper. 


\subsection{The $\Delta$-variance}

The $\Delta$-variance analysis was introduced by Stutzki et al. (1998) and improved and extended by Bensch et al. (2001) and Ossenkopf et al. (2005). Here, we repeat only those definitions that are essential for the centroid analysis.

The $\Delta$-variance in a structure $f(\boldsymbol{x})$ is computed by filtering the data set with a spherically symmetric, normalised wavelet of characteristic size $l$, consisting of a positive inner part and a negative annulus, and computing the variance of the filtered map. Ossenkopf et al. (2005) tested various wavelet shapes, but their mutual differences are not significant for the analysis performed here so that we stick to the ordinary French hat filter from Stutzki et al. (1998). The $\Delta$-variance is then the variance of the filtered map, as a function of the filter size, given by

$\sigma_{\Delta}^{2}(l)=\left\langle\left(f(\boldsymbol{x}) * \bigodot_{l}(\boldsymbol{x})\right)^{2}\right\rangle_{\boldsymbol{x}}$

where the symbol $*$ stands for a convolution, $\bigodot_{l}$ describes the filter wavelet, and the average is taken over the whole data set. If $l$ is the average distance between two points in the core and the annulus in the filter, the $\Delta$-variance spectrum $\sigma_{\Delta}^{2}(l)$ measures the amount of structure on the given scale $l$.

The $\Delta$-variance is related to the power spectrum of a structure $P(\boldsymbol{k})$ by

$\sigma_{\Delta}^{2}(l)=\int P(\boldsymbol{k})\left|\tilde{\bigodot}_{l}(|\boldsymbol{k}|)\right|^{2} d^{n} \boldsymbol{k}$

where $\tilde{\bigodot}_{l}$ is the Fourier transform of the filter function with the size $l$ and $\boldsymbol{k}$ denotes the spatial frequency or wavenumber. In the case of isotropic structures, the power spectrum is spherically symmetric, $P(\boldsymbol{k})=P(|\boldsymbol{k}|)$. This is also the case for the Fouriertransformed filter function as long as it is spherically symmetric in the spatial domain. The power spectrum is given by the Fourier transform of the autocorrelation function

$A(\boldsymbol{l})=\langle f(\boldsymbol{x}) f(\boldsymbol{x}+\boldsymbol{l})\rangle_{\boldsymbol{r}}$.

For power-law power spectra, Stutzki et al. (1998) showed that for $2 \mathrm{D}$ structures in the interval of spectral indices $\beta$ between 0 and 6 , the $\Delta$-variance spectrum is also a power law with the exponent $\alpha=\beta-2$. In three dimensions the range is extended to $0<\beta<7$ and the exponent is $\alpha=\beta-3$. Equivalent slopes are obtained locally in the case of non-power-law power spectra. However, in this case there is no analytic relation for the normalisation factor of the $\Delta$-variance spectrum so that it can only be obtained by numeric integration.

Thus the $\Delta$-variance is basically a very robust method of evaluating the power spectrum of a structure. The advantages of the $\Delta$-variance compared to directly computing the power spectrum result from the smooth filter shape, which provides a very robust way for an angular average independent of gridding effects, and from the insensitivity to edge effects as discussed by Bensch et al. (2001). A possible disadvantage is the implicit radial averaging, which does not allow for seeking signatures of an anisotropy still contained in the 2D power spectrum $P(\boldsymbol{k})$. Such an anisotropy was considered by Esquivel \& Lazarian (2005) but is irrelevant for our studies.

\subsection{Comparing $\Delta$-variance and structure function}

Lazarian \& Esquivel (2003) and Esquivel \& Lazarian (2005) used the (second order) structure function instead of the $\Delta$-variance to characterise the scaling of velocity centroids. The structure function is also related to the autocorrelation function, $D(\boldsymbol{l})=2[A(0)-A(\boldsymbol{l})]$ (see e.g. Miesch \& Bally 1994). With the power spectrum being the Fourier transform of the autocorrelation function, we also have a trivial relation between structure functions and power spectra.

For structures with a power-law power spectrum, Stutzki et al. (1998) analytically studied the relation between the power spectrum, the autocorrelation function and the $\Delta$-variance. They find in the range of spectral indices $3<\beta<5$ in 3D and for $2<\beta<4$ in 2D, and in the limit of infinitely large data sets, power-law structure functions. Using the notation of Lazarian \& Pogosyan (2000), this is the range of steep spectra. Here, the spectral index of the structure function agrees with the index of the $\Delta$-variance spectra discussed above. In the range of shallow spectra with lower power spectral indices, $0<\beta<3$ or $0<\beta<2$ respectively, the autocorrelation function is a power law ${ }^{5}$ so that the structure function must deviate from a power law behaviour. The structure function is always increasing with lag towards the maximum given by twice the total variance of the structure $\sigma_{f}^{2}=A(0)$.

For MHD simulations producing basically steep velocity spectra but with significant deviations from pure power laws Ossenkopf \& Mac Low (2002) compared the centroid velocity structure function with the $\Delta$-variance of the centroid map and showed that both give a similar scaling behaviour, having comparable slopes within a large part of the spectrum., The $\Delta$-variance, however, is advantageous with respect to the detection of pronounced scales in the map and is more robust with respect to observational artifacts. Altogether, the $\Delta$-variance seems to be somewhat better suited to determining the exponent of the power spectrum, as it shows a wider range of power-law behaviour and is stabler with respect to observational restrictions.

On the other hand, Esquivel \& Lazarian (2005) demonstrated that the structure function of centroid velocities can be analytically understood with respect to its composition from density and velocity fluctuations. This represents a clear advantage compared to the $\Delta$-variance. Because of this, we actually performed all tests of the centroid structures reported here both with the $\Delta$-variance analysis and with the structure function. As a surprising result, we find very little difference in the general behaviour. Therefore, we concentrate in the following analysis on the $\Delta$-variance spectra and discuss differences to the structure functions only in Sect. 3.5.

\subsection{Projection effects}

The relation between a 3D structure and projected 2D maps, obtained by integration along the line of sight, has been studied in detail both in terms of the $\Delta$-variance (e.g. Stutzki et al. 1998; Mac Low \& Ossenkopf 2000) and of the structure function (Esquivel \& Lazarian 2005). A projection of the density structure $\rho(\boldsymbol{x}, z)$ is inherently performed when observing the intensity map $I_{\text {int }}(\boldsymbol{x})$ of an optically thin tracer in a medium of constant excitation temperature (Eq. (1)).

The projection effect on the $\Delta$-variance spectrum can be easily understood by realising that the $\Delta$-variance is basically a robust method of deducing the power spectrum. In Fourier space, projection corresponds to the selection of the zero-frequency component in the considered direction. For isotropic structures the power spectral indices of projected maps in any direction agree with the spectral index of the $3 \mathrm{D}$ structure. This is

5 For spatial separations corresponding to wavenumbers smaller than the cut-off wavenumber given by the finite sampling of any system. 
fulfilled by definition for the $\mathrm{fB}$ structures used here for testing. Thus the local slope of the power spectrum $\beta$ is retained, and all components with non-zero spatial frequencies in the considered direction are dropped. Because the $\Delta$-variance is obtained by convolving this power spectrum with the Fourier transform of either a 3D or a 2D wavelet, the resulting spectrum has a local slope $\alpha_{3 \mathrm{D}}=\beta-3$ or $\alpha_{2 \mathrm{D}}=\beta-2$, respectively. The mutual translation is straightforward. The exponent of the power spectrum is retained on projection, while the index of the $\Delta$-variance ${ }^{6}$ is increased by one. This was confirmed in the application of the $\Delta$-variance analysis to the 3D density structure of (magneto-)hydrodynamic simulations and their projection onto maps by Mac Low \& Ossenkopf (2000).

For power-law power spectra, the translation of the amplitudes can also be performed analytically following the formalism provided in the Appendix of Stutzki et al. (1998). As an approximation, we can also use the simple empirical relation

$\sigma_{\Delta, 3 \mathrm{D}}^{2}(l)=\sigma_{\Delta, 2 \mathrm{D}}^{2}(l) \times \frac{l}{l_{\text {cube }}} \times 1.97 \exp \left(-\frac{\beta}{2.83}\right)$,

which is accurate within a few percent for power spectral indices $\beta$ between 1 and 4 and cube sizes of at least $32^{3}$ pixels. Even for sufficiently smooth, but non-power-law $\Delta$-variance spectra, Eq. (9) can be applied by using an index $\beta(l)$ derived from the local slope.

A general problem is, however, the actual loss of information by projection. There is no way to recover the Fourier amplitudes that are dropped by the projection. Thus the re-translation from the $2 \mathrm{D} \Delta$-variance spectrum into the corresponding $3 \mathrm{D}$ spectrum is only possible by assuming isotropy. Mac Low \& Ossenkopf (2000) and Ossenkopf \& Mac Low (2002) studied the degree of anisotropy in hydrodynamic and magneto-hydrodynamic simulations by comparing $2 \mathrm{D}$ and $3 \mathrm{D} \Delta$-variance spectra and found that the assumption is clearly violated for simulations with strong magnetic fields but reasonably justified for most other simulations.

Figure 3 demonstrates the influence of the projection effects on the $\Delta$-variance spectra of two fBm's. The upper graph represents an $\mathrm{fBm}$ structure with a shallow index $\beta=2.6$ and the lower graph a steep spectrum with $\beta=3$.7. The $\Delta$-variance spectra measured in 3D and for the projected structure follow the theoretical power-law relation almost exactly with the exponents $\alpha=\beta-3$ or $\alpha=\beta-2$, respectively. The triangles stand for the results from the $\Delta$-variance computed in $3 \mathrm{D}$ and translated into a 2D spectrum using Eq. (9). We find an excellent agreement with the spectra obtained directly from the projected maps.

Beyond the plotted range, the $\Delta$-variance spectra show a turn-over at about half of the total size of the simulated cube arising from the lack of larger structures due to the periodicity condition in the construction of the data (see Bensch et al. 2001). Because of the loss of significance at large lags, the spectra are only computed up to lags of about a third of the cube size.

\section{Centroid composition effects}

When taking their relation to the autocorrelation function, both the $\Delta$-variance and the structure function of velocity centroid maps will be given by averages of the products $v_{\mathrm{c}}(\boldsymbol{x}) v_{\mathrm{c}}(\boldsymbol{x}+\boldsymbol{l})$ (see Eq. (8)). Using the decomposition of the velocity centroids in Eq. (5) and assuming a zero average velocity $v_{0}$, we see that

${ }^{6}$ The same applies to the structure function, but in a limited spectral range.
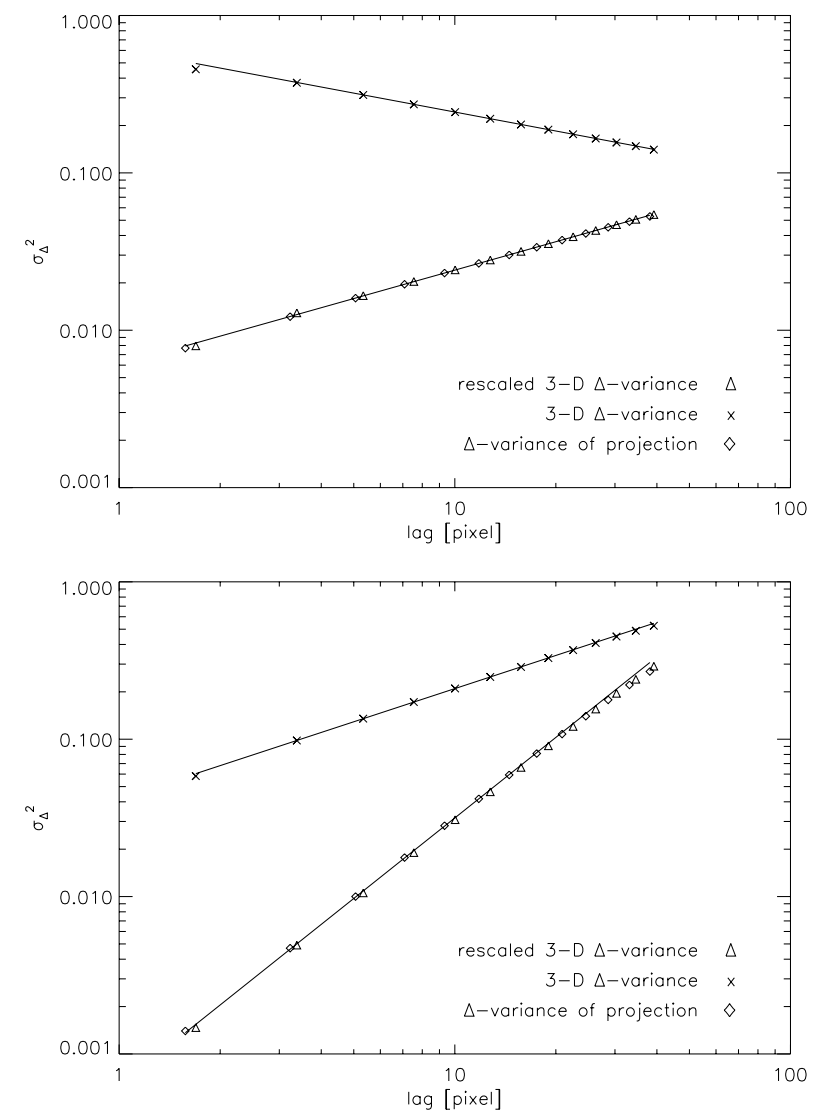

Fig. 3. $\Delta$-variance spectra determined in 3D structures and their projection together with the translation of the $3 \mathrm{D} \Delta$-variance spectrum into the corresponding 2D spectrum using Eq. (9). For the upper plot, an fBm with $\beta=2.6$ shifted by $2 \sigma$ and truncated at zero was used. The lower plot shows the result for an $\mathrm{fBm}$ with $\beta=3.7$. The solid lines mark slopes corresponding to the spectral indices $\beta-3$ and $\beta-2$.

four terms characterise the scaling:

$$
\begin{aligned}
& A_{v_{\mathrm{c}}}(\boldsymbol{l})=\rho_{0}^{2}\left\langle\int \mathrm{d} z \delta v(\boldsymbol{x}, z) \times \int \mathrm{d} z \delta v(\boldsymbol{x}+\boldsymbol{l}, z)\right\rangle_{\boldsymbol{x}} \\
& +\left\langle\int \mathrm{d} z \delta \rho(\boldsymbol{x}, z) \delta v(\boldsymbol{x}, z) \times \int \mathrm{d} z \delta \rho(\boldsymbol{x}+\boldsymbol{l}, z) \delta v(\boldsymbol{x}+\boldsymbol{l}, z)\right\rangle_{\boldsymbol{x}} \\
& +\rho_{0}\left\langle\int \mathrm{d} z \delta v(\boldsymbol{x}, z) \times \int \mathrm{d} z \delta \rho(\boldsymbol{x}+\boldsymbol{l}, z) \delta v(\boldsymbol{x}+\boldsymbol{l}, z)\right\rangle_{\boldsymbol{x}} \\
& +\rho_{0}\left\langle\int \mathrm{d} z \delta \rho(\boldsymbol{x}, z) \delta v(\boldsymbol{x}, z) \times \int \mathrm{d} z \delta v(\boldsymbol{x}+\boldsymbol{l}, z)\right\rangle_{\boldsymbol{x}} .
\end{aligned}
$$

The first term is the autocorrelation function of the projected velocity fluctuations. If this term dominates, the scaling behaviour of the centroid velocities reflects the scaling behaviour of the velocity structure exactly. In this case it is easy to deduce the properties of the velocity structure from an observed map of centroids. We find the simple projection of the velocity structure onto a $2 \mathrm{D}$ map as in the case of the column density map reflecting the 3D density structure. The second term describes a combination of the fluctuations of the density and the velocity structure. The term also contains the mutual correlation between density and velocity fluctuations along the line of sight. The third and fourth terms quantify the cross-correlation between velocity fluctuations at one point and density fluctuations at another point. In case of isotropic media, both terms are identical. They should statistically vanish in the case of independent density and 
velocity structures, but some remainders due to accidental crosscorrelations are expected for any particular realisation. A similar decomposition in terms of the structure function was provided by Lazarian \& Esquivel (2003). From the decomposition in Eq. (10), we see that the ratio between the average density and the density fluctuations should provide a criterion for deciding whether the centroid map is a good measure of the scaling of the velocity field. In the following we test the composition of centroid velocity maps from fBm structures by adjusting their parameters in such a way that we cover the full range of observed spectral indices for the density and velocity structure in the interstellar medium.

\subsection{The density zero level}

A major problem with the artificial simulation of density structures is the mutual incompatibility of Gaussian fluctuations and strictly positive values for the density. As discussed in Sect. 2.2, fBm structures always show a Gaussian distribution of values. Moreover, the analytic expressions for the velocity centroids derived by Lazarian \& Esquivel (2003) are also based on the assumption of Gaussian fluctuations. However, as long as the average of a Gaussian distribution is not large compared its dispersion, negative values are unavoidable for sufficiently large samples.

A common way to create positive densities is to add a constant density until the minimum value in the cube falls to zero (Miville-Deschênes et al. 2003a; Esquivel et al. 2003). A major drawback of this method is, however, that the minimum value of a Gaussian distribution depends on the exact realisation of the random numbers used to generate the distribution, and it is very sensitive to the size of the data cube. Thus the added value, then providing the average density $\rho_{0}$, may significantly vary from simulation to simulation. By renormalising the average density to unity as proposed by Miville-Deschênes et al. (2003a) and Esquivel et al. (2003), the variation is only transferred to the standard deviation of the density distribution because the ratio between standard deviation and mean is retained. Moreover, the approach results typically in $\sigma_{\rho} /\langle\rho\rangle<0.3$ (Miville-Deschênes et al. 2003a). Such values contradict many observational data (see e.g. Jenkins 2004). Density fluctuations with $\delta \rho / \rho \approx 1$ are expected for Mach numbers approaching unity. Such Mach numbers characterise warm media, while colder parts of the ISM tend to have supersonic velocities (see Elmegreen \& Scalo 2004) leading to even larger density fluctuations (Falgarone et al. 1998; Padoan et al. 1997).

To a certain extent these problems can be circumvented by combining the density shift with a truncation of the residual negative tail. When we shift the density distribution, e.g. by $\rho_{0}=1 \sigma_{\rho}$ by adding this constant value, and discard all points falling below zero, only $8 \%$ of the points from the original distribution are set to a zero value so that the statistical properties of the overall structure are hardly changed. In this way we can obtain positive densities and a $\sigma_{\rho} /\langle\rho\rangle$ ratio of about one, avoiding all problems from a dependency on the resolution and on the exact random numbers. One has to keep in mind, however, that the truncation of the density structure can have a noticeable influence on the scaling properties. The pure addition of the constant density does not affect affect the spectrum because it is scale-independent.

To test the possible error introduced by truncating the distribution at a given density level, we analysed the truncated fBm's and compared them to the original spectra. The result is shown for a spectral index $\beta=2.6$ and different truncation levels in

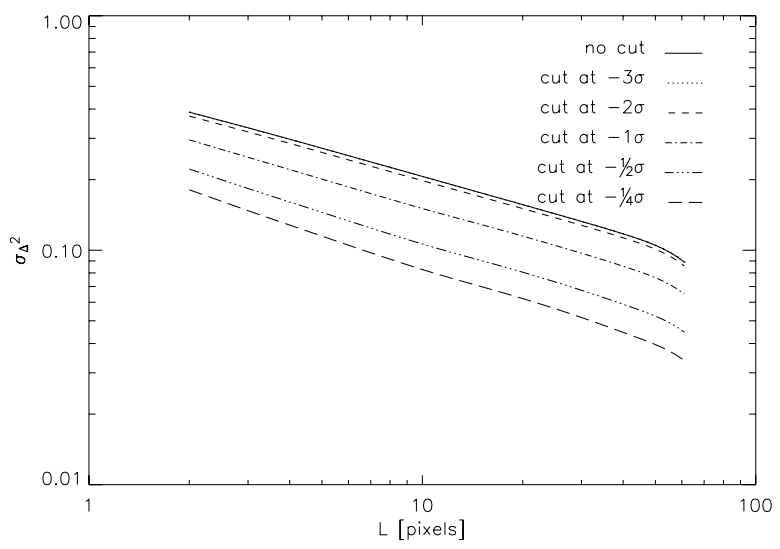

Fig. 4. $\Delta$-variance spectra of $3 \mathrm{D}$ density structures obtained by shiftand-truncate from an $\mathrm{fBm}$ with $\beta=2.6$ and different truncation levels. The spectrum for the density cut at $3 \sigma$ is partially indistinguishable from the original spectrum.

Fig. 4. The actual shift of the density by $\rho_{0}$ does not influence these spectra because the $\Delta$-variance is insensitive to any constant offset. We see the pure truncation effect. For truncation levels of $2 \sigma_{\rho}$ and above, the spectra are practically not changed. For truncation levels between $0.5 \sigma$ and $2 \sigma$, the shape of the spectra is retained but shifted to lower absolute values. This can be explained by the reduction of the total variance in the data cubes, which is visible in the scale-dependent $\Delta$-variance as well. The original distribution was normalised to a variance of unity in this example, whereas the truncation leads to reduced variances of $0.96,0.76$, and 0.56 for the $2 \sigma, 1 \sigma$, and $0.5 \sigma$ truncation levels, respectively. These are exactly the numbers by which the $\Delta$-variance spectra in Fig. 4 are shifted relative to the original spectrum. Only for a truncation level at $1 / 4 \sigma$ is the slope of the spectrum changed; i.e. the scaling behaviour of the structure is modified. In this case the absolute shift of the $\Delta$-variance spectrum also no longer matches the corresponding reduction of the total variance of the density distribution relative to the original value.

Examining the resulting projected maps shows that the relation between the $3 \mathrm{D}$ scaling and the $2 \mathrm{D}$ scaling given in Eq. (9) is also preserved down to truncation levels of $0.5 \sigma$. Corresponding studies for different spectral indices show that the $\Delta$-variance spectra are least sensitive to truncations at low spectral indices, between 2 and 2.5, where even truncation levels of $0.25 \sigma$ do not change the scaling behaviour and the relation between total variance and $\Delta$-variance. At spectral indices close to four, in contrast, the $0.5 \sigma$ truncation plot shows already significant deviations, so that we conclude that a negligible statistical impact on the scaling behaviour is only guaranteed at truncation levels around $1 \sigma$ and above. The shift-and-truncate method to create positive densities is thus not perfect in terms of retaining the original scaling properties of the structure, but the introduced deviations are still small compared to those introduced by the non-linear transformations discussed in Sect. 2.2. They would be hardly detectable in observed data, although we have to take them into account when performing a detailed quantitative analysis.

Figure 5 shows two actual examples for the influence of the density zero level definition on the measured centroid velocity spectra. The scaling behaviour of the centroids was computed in terms of the $\Delta$-variance spectra for three different shift-andtruncate levels of fBm generated density structures. To judge how far they reflect the original density or velocity structure, 

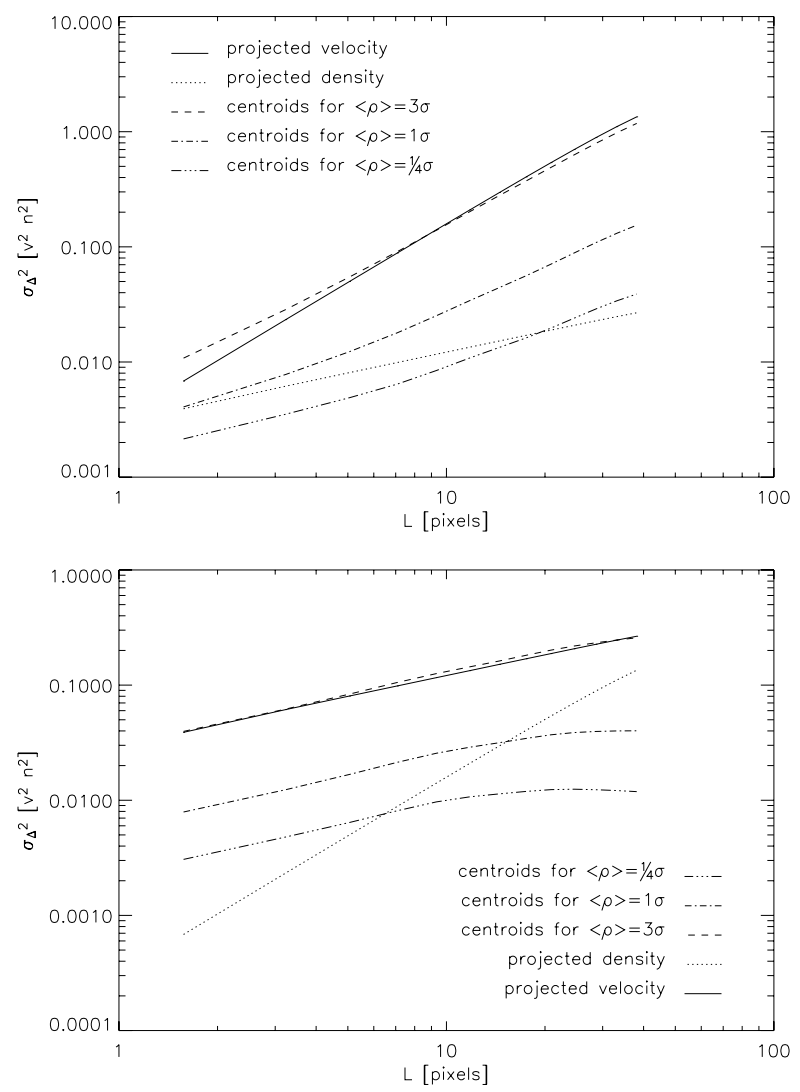

Fig. 5. Comparison of the $\Delta$-variance spectra of the weighted centroid velocities with the spectra of the original density and velocity structure for different truncation levels. To plot equivalent quantities, the projected density is multiplied by $\left\langle v^{2}\right\rangle$ and the projected velocity by $\left\langle\rho^{2}\right\rangle$ (see Esquivel \& Lazarian 2005). The $\left\langle\rho^{2}\right\rangle$ factor was computed for the $3 \sigma$-cut density cube. The corresponding plots for $1 \sigma$ and $0.25 \sigma$ would be shifted down by a factor 5.2 and 13.5 , respectively. The upper plot was computed from an $\mathrm{fBm}$ density structure with $\beta=2.6$ and a velocity structure with $\beta=3.7$, while the lower plot used the opposite spectral indices.

we have also plotted the $\Delta$-variance spectra of these projected quantities multiplied with the mean square of the complementary quantity to guarantee units equivalent to the centroids.

The upper plot shows the combination of a shallow density spectrum with a steep velocity spectrum, matching a situation that is typically observed in molecular clouds (see Sect. 2.2). The absolute shift of the curves for weighted centroids is mainly determined by the different values of $\left\langle\rho^{2}\right\rangle$ produced by different average densities. However, this shift does not influence the characteristic scaling behaviour within the structure. Looking at the slopes of the centroid spectra, we find a confirmation of the general considerations on the role of the density zero level $\rho_{0}$ given above. If the density structure is dominated by a large average, i.e. in the case of $\rho_{0}=3 \sigma_{\rho}$, the centroid velocities are basically given by a projection of the velocity structure, so that they reproduce the original velocity scaling behaviour. For lower average densities, i.e. a lower relative contribution of the pure velocity projection given by the first term in Eq. (10), the centroid scaling becomes shallower with an exponent that is close to that of the velocity structure on large scales and an exponent close to that of the density structure on very small scales and the lowest values of $\rho_{0}$. This plot seems to confirm the transition from purely velocity-dominated centroids to density-dominated centroids as originally interpreted by Lazarian \& Esquivel (2003).
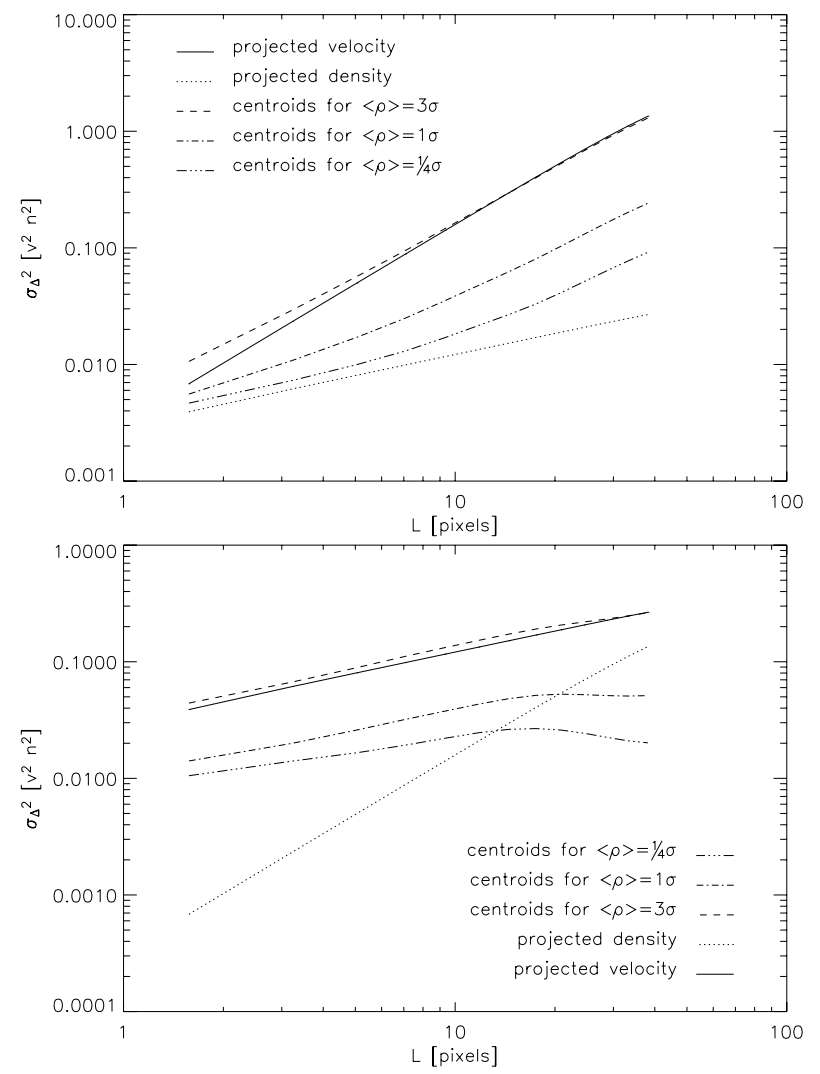

Fig. 6. Same as Fig. 5 but for normalised centroids. They are rescaled by the factor $\left\langle\rho^{2}\right\rangle$ to obtain comparable dimensions.

If we consider, however, the opposite situation of spectral indices in the lower plot, we only find that the centroid scaling becomes less and less representative for the actual velocity structure when reducing the average density $\rho_{0}$. Their scaling does not tend towards the scaling of the column density structure but becomes shallower as well. This fact is confirmed in all simulations with other combinations of spectral indices. At low values of $\sigma_{\rho} / \rho_{0}$, the centroids match the projected velocity structure, whereas their scaling becomes shallower for lower average densities irrespective of the actual spectral index of the density structure. The spectral index of the density structure determines, however, on which scales the deviations occur. For density structures with a shallow spectral index, i.e. dominated by many small-scale fluctuations, the main effect occurs on small scales. In contrast, we find the main deviations at large scales, when the density structure has a steep index, representing a relative dominance of large-scale fluctuations. When interpreting changes in the slope of the $\Delta$-variance spectrum of observed centroid maps, we can thus use the known information on the projected density scaling to judge whether they represent an actual deviation of the velocity structure from self-similarity or whether they might be produced just by the centroid composition effects. In general, we find a bigger impact on the overall centroid spectrum as both spectra become shallower. However, it is not clear that the a shallow velocity field is physically motivated (Esquivel \& Lazarian 2005).

In Fig. 6 we show the same effects for the ordinary, normalised centroids instead of the weighted centroids used in Fig. 5. We find the same general behaviour as for the weighted centroids but differences in details. In all cases with high average densities, i.e. for $\sigma_{\rho} / \rho_{0}<1$, the normalised centroids provide a slightly better reproduction of the original velocity structure than 
do the weighted centroids. At lower densities, they are somewhat less changed for shallow density spectra and somewhat more changed for steep density spectra. The modifications correspond approximately to the same effect that a change of $\sigma_{\rho} / \rho_{0}$ by a factor 1.5 would have for the weighted centroids. In general we can state, however, that either both centroid definitions reveal the true velocity structure or none of them do. The direct retrieval of the velocity scaling from the $\Delta$-variance spectra of the centroids will only succeed when the average cloud density is significantly larger than the density dispersion. In these cases the normalised centroids are marginally better than the weighted centroids.

These results explain the differences and agreements between the previous studies on velocity centroids discussed so far in the literature. The studies of Miville-Deschênes et al. (2003a) and Lazarian \& Esquivel (2003) used a relatively large average density and they indicated a good match between centroid spectra and projected velocity spectra. The mechanism of producing positive densities from fBm's by adding large constant values used by Miville-Deschênes et al. (2003a) and Esquivel et al. (2003) gave results that correspond to our results for applying the shift-and-truncate technique with a large average density, i.e. when we add the $3 \sigma$ density offset. Both centroid definitions follow the actual velocity scaling over a wide range of scales, deviating at most at the very ends of the spectra in this case. One has to emphasise that this matching is only produced by adding a large $\rho_{0}$ value, so that the general conclusion that centroids are a good measure of the velocity structure drawn by Miville-Deschênes et al. (2003a) and applied to interpreting observational data by Miville-Deschênes et al. (2003b) does not hold for the general case of interstellar gas with substantial density fluctuations.

In cases with lower average densities, all using a combination of steep velocity spectra with shallow density spectra, Ossenkopf \& Mac Low (2002), Lazarian \& Esquivel (2003), and Brunt \& Mac Low (2004) found centroid spectra that were shallower than the velocity spectrum. The hydrodynamic and magneto-hydrodynamic turbulence models studied by Ossenkopf \& Mac Low (2002) were characterised by steepvelocity spectra with $\beta_{v} \approx 4.0$, shallow-density spectra with $\beta_{\rho} \approx 2.5 \ldots 2.7$, and a high-density contrast with $\sigma_{\rho} / \rho_{0}>5$ thus corresponding closely to the conditions for the low-density curve in the upper plot of Fig. 6. With a limited dynamic range for fitting the $\Delta$-variance spectra, it is obvious that the flattening of the centroid spectra relative to the original velocity spectrum seen in the figure can be misinterpreted as a constant reduction of the slope by one.

Our results can also explain the findings of Brunt \& Mac Low (2004). They studied the characteristics of velocity centroids of HD and MHD turbulence simulations as a function of Mach number. With the known relation between Mach number and density dispersion (Padoan et al. 1997), their finding of a growing discrepancy between the average spectral index of the velocity distribution and of the centroid map with growing Mach number can be explained by the impact of an increasing $\sigma_{\rho} / \rho_{0}$ ratio, which reduces the relative contribution of the projection term. This is most clearly seen in the models of decaying turbulence where, e.g., for an initial $\sigma_{\rho} / \rho_{0}$ ratio of 1.1, the centroid spectrum is shallower by 0.8 than the velocity spectrum, whereas it is only shallower by 0.1 for the final $\sigma_{\rho} / \rho_{0}$ ratio of 0.5. We have to emphasise, however, that this approach cannot explain the differences in the spectral indices obtained by Brunt \& Mac Low (2004) for MHD models observed perpendicular or
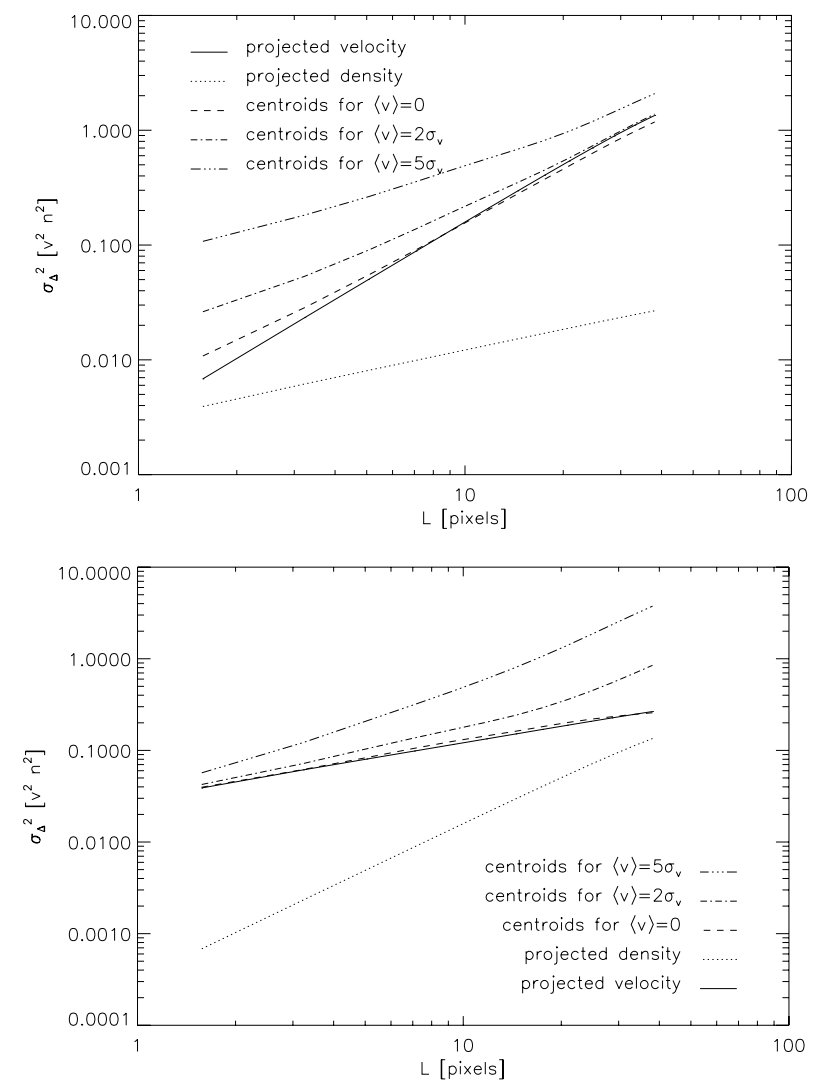

Fig. 7. $\Delta$-variance spectra of the centroid velocities computed using the density and velocity structures from Fig. 5 and different $v_{0}$ levels. A density structure with $\langle\rho\rangle=3 \sigma_{\rho}$ was used here.

parallel to the main magnetic field direction. In these cases, the isotropy assumption used in our analysis is clearly violated.

\subsection{The velocity zero level}

The composition of weighted centroids (Eq. (5)) is a priori symmetric with respect to density and velocity. In the decomposition in Eq. (10), we have assumed, however, that the velocity scale is chosen in such way that $v_{0}=0$ while $\rho_{0}>0$. To better understand the centroid behaviour, it is useful to perform an experiment using velocity fields with $v_{0}>0$. From the symmetry of the problem, we expect to find a centroid behaviour matching the density scaling for large average velocities $v_{0}$ in the same way as we find centroids matching the velocity scaling for large average densities $\rho_{0}$. Indeed, we obtain the new term $v_{0}^{2}\left\langle\int \mathrm{d} z \delta \rho(\boldsymbol{x}) \times \int \mathrm{d} z \delta \rho(\boldsymbol{x}+\boldsymbol{l})\right\rangle_{\boldsymbol{x}}$ in Eq. (10) if $v_{0} \neq 0$. It contains the spectrum of the projected density fluctuations. In contrast to the density treatment, we do not apply any truncation to the velocity structure when shifting it to $v_{0}>0$. The experiment thus provides an additional test for the significance of the truncation. If the simple shift of the velocity structure behaves the same as the shift-and-truncate of the density structure, we can be sure that all effects result from the selection of the average values and not from the truncation.

Figure 7 shows the impact of different velocity offsets on the centroid $\Delta$-variance spectra. A high average density, $\rho_{0}=3 \sigma_{\rho}$, was chosen to guarantee that the centroid spectrum for $v_{0}=0$ is dominated by the velocity structure. The curves for $v_{0}=0$ are identical to the $\rho_{0}=3 \sigma_{\rho}$ curves in Fig. 5. When increasing the average velocity, we find a transition to shallower spectra in 
the upper plot similar to the effect of a reduced average density in Fig. 5. The slope of the centroid spectrum remains close to the slope of the velocity spectrum on large scales and on small scales it takes the slope of the column density spectrum. In the lower panel we also find that the centroid spectrum is more and more similar to the column density spectrum when increasing the average velocity. This is opposite to the effect of reducing the average density in the lower panel of Fig. 5. The adjustment of the average velocity reproduces the transition from velocitydominated spectra to density-dominated spectra, as predicted by Lazarian \& Esquivel (2003).

The equivalence in the impact of the velocity shift on the centroid spectra to the impact of the shift-and-truncate method for the density structures proves that the main change of the centroid spectrum is due to the added offsets and not due to the truncation of the density structure at its low density wing. Unfortunately, the numerical experiment cannot be exploited to derive the true velocity scaling when the average density is so small that the centroid spectra for $v_{0}=0$ are "density-contaminated". By increasing $v_{0}$ we will only increase the contribution from the density scaling, which is already known from the projected intensity maps, but we cannot remove the effect of the combination of density and velocity fluctuations.

\subsection{Further decomposition}

The results obtained so far show that the density zero level basically changes the contribution from the first term in Eq. (10) representing the pure projection of the velocity structure. In a next step we investigate the relative contribution of the other three terms to the deviation measured between the projected velocity spectra and the centroid spectra. The third and fourth terms vanish if there is no cross-correlation between the density and velocity fields. This should be the case for our independently generated fBm structures. We expect, however, that in every realisation some accidental correlations occur, so that the two terms are only negligible in the ensemble average.

If the density field is known, we can obtain the second term, i.e. the combination of density and velocity fluctuations, by constructing an auxiliary density field $\rho_{\text {aux }}=\rho-\rho_{0}$ and computing the weighted centroids for this auxiliary quantity. As the average density of the auxiliary field vanishes, the derived centroids directly match the second term in Eq. (10). This procedure is illustrated in Fig. 8 where we plot the centroid spectrum for the auxiliary field $\rho_{\text {aux }}$, and compare the full centroid spectrum obtained from the original density structure with the sum of this second term and the pure velocity scaling term. For the sake of comparison we also plot the spectrum of the projected density and velocity structure, where the velocity spectrum is multiplied here by $\rho_{0}^{2}$ to represent the first term in Eq. (10) exactly. The same combination of spectral indices as used in Fig. 5 was taken. A $0.25 \sigma_{\rho}$ shift-and-truncate level was used for the density structure, so that the centroid spectrum deviates considerably from the spectrum of the velocity fluctuations.

For all the combinations of spectral indices studied, we obtained a good match between the sum of the projected velocity spectrum and the centroid spectrum from the auxiliary field of density fluctuations with the full spectrum of the velocity centroids. Nevertheless, we always found a non-negligible difference between the two curves, resulting from the accidental crosscorrelations contributing to the third and fourth term, which are not contained in the sum. We also found that the second term, giving the combination of all fluctuations, has a spectrum which is always shallower than either of the projected spectra involved.
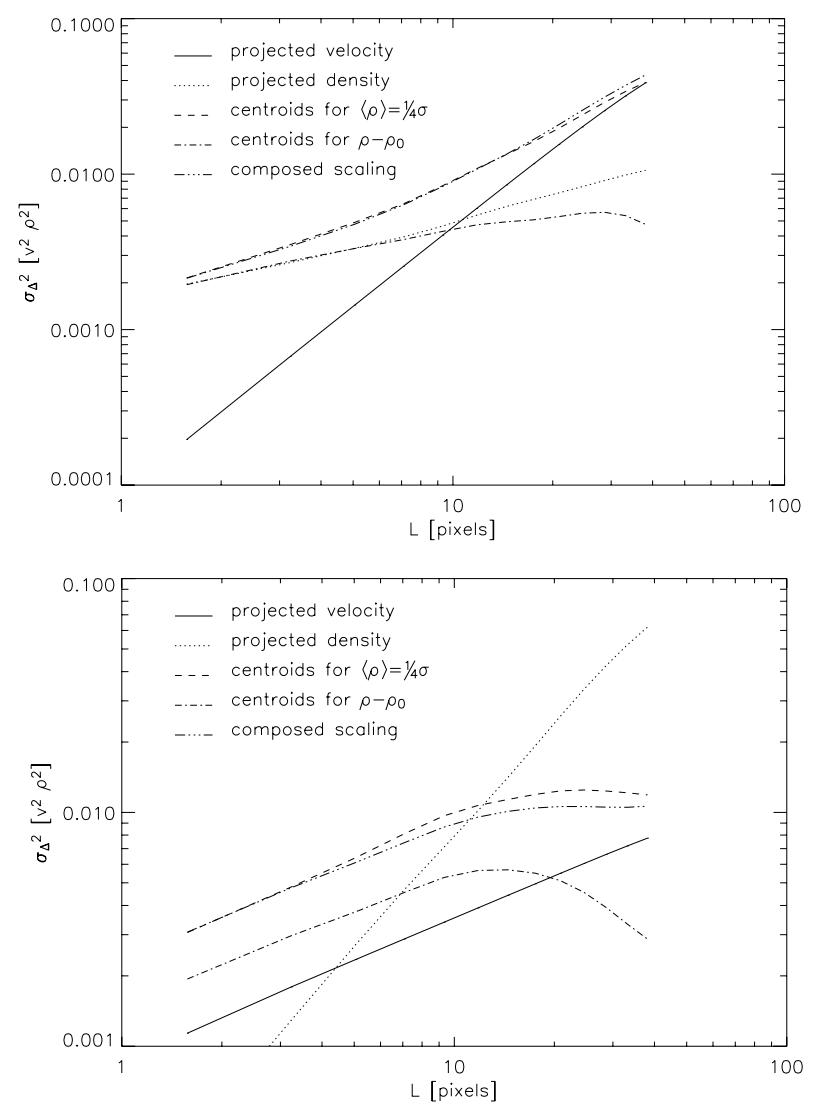

Fig. 8. Decomposition of the $\Delta$-variance spectra of the weighted centroid velocities for the density and velocity structures from Fig. 5 using shift-and-truncate by $1 / 4$ of the original standard deviation for the density structure. The solid line represents the contribution from the velocity structure projected with a $\rho_{0}$ weighting and the dash-dot line represents the centroid contribution from the auxiliary density fluctuation field. The sum of both terms (dash-dot-dot-dot) is very close to the measured centroid spectrum (dashed).

For a steep spectrum of density fluctuations, its slope even turns negative at large scales. This explains why the total spectrum of the weighted centroids is always shallower than the projected velocity spectrum, independent of the spectral index of the density spectrum.

The computations have confirmed the theoretical expectation that the spectrum of velocity centroids consists of only two main contributions: the pure projection of the velocity structure determined by the average density $\rho_{0}$ and a shallow term mainly determined by the density fluctuations. In Sect. 4 we show how this decomposition can be exploited to measure the actual velocity structure from observed centroid maps if the $\sigma_{\rho} / \rho_{0}$ ratio can be estimated independently.

\subsection{Matching criteria}

We have seen that the single quantity giving the ratio between the strength of the density fluctuations and the average density $\sigma_{\rho} / \rho_{0}$ is able to discriminate between the different behaviours of the centroid spectra. For low values of this ratio, the spectra are dominated by the actual velocity structure so that the $3 \mathrm{D}$ velocity scaling is preserved in observed centroid maps. For $\sigma_{\rho} / \rho_{0} \lesssim 0.5$ the $\Delta$-variance spectrum of the centroid map directly measures the spectral index of the underlying velocity structure. For higher values, the centroid spectra are 
always shallower than the spectra from the projected velocity maps. They are produced by a combination of density and velocity fluctuations.

In contrast to the suggestion of a density-dominated regime by Lazarian \& Esquivel (2003), the systematic study of a wide range of combinations of spectral indices with the $\Delta$-variance spectra shows no indications of a transition from velocitydominated centroids to density-dominated centroids, but rather a transition to "density-contaminated" spectra. Using a decomposition of structure functions similar to Eq. (10), Lazarian \& Esquivel (2003) identified a term that indeed traces density fluctuations. They showed that in general centroids do not trace the velocity fluctuations directly. However, in their numerical tests, they used a combination of steep velocity and shallow density spectra, and disregard a cross term that is equivalent to the convolution of velocity and density fluctuations presented here. The shallow centroid spectrum was interpreted as the density spectrum. We have demonstrated that for the $\Delta$-variance spectra a density-dominated regime arises only if we choose a velocity scale with an offset so that $v_{0} \neq 0$. However, the combination of the facts that the centroid spectrum is always shallower than the velocity spectrum and that most observed density spectra are shallower as well can give the false impression that centroids trace the density scaling for large ratios $\sigma_{\rho} \rho_{0}$.

Esquivel \& Lazarian (2005) presented another criterion for a match between centroid and velocity scalings, namely, $X^{2} \sigma_{v_{\mathrm{c}}}^{2} \gg\left\langle v^{2}\right\rangle \sigma_{I_{\text {int }}}^{2}$. They stated, however, that it is not clear how large the ratio $X^{2} \sigma_{v_{\mathrm{c}}}^{2} /\left(\left\langle v^{2}\right\rangle \sigma_{I_{\text {int }}}^{2}\right)$ should eventually be to guarantee that the centroids reliably represent the velocity structure. When applied to the overall data cubes, we find that the distinctive power of the criterion is limited. In the examples plotted above, we obtain for instance a ratio of 35 when using the $3 \sigma$ shift-and-truncate level of the density distribution and a ratio of 3.0 for the $0.25 \sigma$ shift-and-truncate level in the case of the shallow density and steep velocity spectrum. In contrast, we obtain corresponding ratios of 2.6 and 0.32 , respectively, for the combination of steep density and shallow velocity spectrum. In both cases the $3 \sigma$ shift-and-truncate level gives a good match between centroid and velocity scaling while the $0.25 \sigma$ level results in very poor agreement. Thus the global criterion is poorly quantified.

The criterion can be rewritten in a scale-dependent form (Lazarian \& Esquivel 2003): $X^{2} D_{v_{\mathrm{c}}}(\boldsymbol{l}) /\left(\left\langle v^{2}\right\rangle D_{I_{\mathrm{int}}}(\boldsymbol{l})\right) \gg 1$, when we consider the structure function of the two maps at a given lag $l$. One might assume that this criterion should hold as well for $\Delta$-variance spectra because of their similar scaling properties. Then a ratio $X^{2} \sigma_{\Delta, v_{\mathrm{c}}}^{2}(l) /\left(\left\langle v^{2}\right\rangle \sigma_{\Delta, I_{\text {int }}}^{2}(l)\right)$ much larger than unity indicates a good match of the centroid $\Delta$-variance spectrum with the true velocity spectrum. The denominator grows compared to the numerator with increasing scales when the density spectrum is steeper than the velocity spectrum. In this case, matched by the lower panels of Figs. 5-8, the largest deviations in the centroid spectrum from the velocity spectrum should occur on large scales, whereas the slopes of the $\Delta$-variance spectra should match on small scales. This is indeed the behaviour that we observe in these figures. For the opposite relation of spectral indices, where the velocity spectrum is steeper than the density spectrum, as seen in the upper panels of the figures, the ratio is growing towards larger scales, and in fact we find the best matches of the scaling behaviour on large scales and the main deviation on small scales.

Using the $\Delta$-variance spectra in Fig. 5, we can evaluate the criterion by eye from the plots. When the curves for the centroids fall well above the dotted line giving the density spectrum, the centroid spectrum should be a reliable tracer of the velocity structure. The same test can be performed in the analysis of observed data, because the $\Delta$-variance spectra of the intensity and the centroid velocity maps and the average velocity dispersion are easily measured in observed line data. However, we find that the actual significance is also limited. In the upper panel of Fig. 5 with the shallow density and steep velocity spectrum, we find that the velocity spectrum is reproduced by the centroid spectrum when the centroid $\Delta$-variance exceeds the values from the column-density structure by about a factor four, whereas for the steep density and shallow velocity structure we get a good match even if the centroid curve falls just above the column density spectrum. For other combinations of spectral indices, we find that a ratio of two is sufficient to guarantee a match between centroid spectrum and velocity spectrum, as long as the density spectrum is very steep $\left(\beta_{\rho}>3.5\right)$, whereas ratios as high as 100 may be required to guarantee a match when the density spectrum has an index shallower than 2.5. When using the normalised centroids in Fig. 6 we cannot derive an equivalent criterion to estimate the match between centroid scaling and velocity scaling based on the measured map spectra. This is a clear practical advantage of the weighted centroids.

Thus we can basically confirm the criterion, when applied in its scale-dependent form to $\Delta$-variance spectra, but have to emphasise that there is no single value for the ratio where the transition between velocity-dominated and "density-contaminated" behaviour appears, but that the exact shape of the density spectrum has to be taken into account.

\subsection{Comparison of $\Delta$-variance spectra and structure function}

As the structure function is related to the autocorrelation function, the decomposition in Eq. (10) also applies to the contributions to the structure function. Lazarian \& Esquivel (2003), however, showed that the second term representing the combination of density and velocity fluctuations can be further split into two separate contributions in terms of the structure function. As one of them represents the pure density fluctuations, they suggested that the structure function can undergo a transition from a velocity-dominated spectrum to a density-dominated spectrum.

To test this behaviour we repeated the experiments shown in Figs. 5 to 7 for structure functions. In general we expect to see clear deviations from power-laws as the projection of structure functions results always in broken power laws. Structure functions of 2D projections can be represented by two asymptotic power laws: one at small lags $\left(|\boldsymbol{l}| \ll z_{\text {tot }}\right)$ having a spectral index $\beta-2$ for both shallow and steep spectra, and another one at large lags $\left(|\boldsymbol{l}| \gg z_{\text {tot }}\right)$ with a spectral index $\beta-3$ for steep spectra and 0 (constant) for shallow spectra (Esquivel \& Lazarian 2005). Taking the general limitation of a restricted dynamic range of scales, both in the fBm simulations and in most observed maps, the 2D structure functions will always fall in the transition between the two asymptotes so that their slope cannot be reconciled directly, preventing a direct recovery of the underlying 3D statistics. Hence, no simple inversion of the projection problem is possible. Compared to the $\Delta$-variance spectra, the spectra of structure functions are thus always somewhat more curved with steeper slopes at small lags and shallower slopes at large lags, but in spite of the different analytic decomposition of the structure function of centroid velocities demonstrated 
by Esquivel \& Lazarian (2005), the general behaviour is always very similar to the $\Delta$-variance spectra.

The measured changes due to variations in the density and velocity zero level are also almost identical to the behaviour shown in Figs. 5 to 7. For the centroids obtained from the density structure with the $3 \sigma_{\rho}$ shift-and-truncate level, we find a very good match between the structure functions of the centroids and the projected velocity structure. The spectra flatten, however, if the density dispersion is in the order of the average density. Main deviations occur on large scales when the density spectrum is steep and on small scales when it is shallow. When comparing $\Delta$-variance spectra and structure functions in detail, we find that the centroid structure functions resemble the true velocity structure always slightly better than the $\Delta$-variance spectra. This might be partially due to the somewhat lower sensitivity of the structure function to rapid changes in the power spectrum as found by Ossenkopf \& Mac Low (2002), but might also indicate a slight advantage of the structure function compared to the $\Delta$-variance spectra when applied to centroid maps.

There is again no transition from velocity-matching behaviour to density-matching behaviour, but rather a densitycontaminated structure with a spectrum that is shallower than the true velocity spectrum. We also find a confirmation of the scaledependent criterion of Esquivel \& Lazarian (2005) for a match between centroid spectrum and true velocity spectrum. Here, the critical ratio $X^{2} D_{v_{\mathrm{c}}}(\boldsymbol{l}) /\left(\left\langle v^{2}\right\rangle D_{I_{\mathrm{int}}}(\boldsymbol{l})\right)$ for a match between centroid structure function and velocity structure function for a particular combination of spectral indices is always somewhat smaller compared to the $\Delta$-variance spectra. For steep density spectra, a ratio of one always seems to be sufficient, whereas a ratio of 20 may be required for shallow density spectra to guarantee a velocity-dominated centroid behaviour.

\section{Derivation of the velocity structure from density-contaminated centroids}

Whenever the average density of the medium is too small, such that the centroid spectrum no longer reflects the underlying velocity spectrum, we can deduce the true velocity spectrum from measured centroids only when we find a way to compute the second term in Eq. (10) and when the last two terms produced by the accidental correlations are negligible. Based on the results of the decomposition shown in Fig. 8, we propose an iteration scheme which computes the second term (the convolution of the two fluctuation spectra) from the first term (the velocity projection weighted with $\rho_{0}^{2}$ ) obtained in a previous iteration step, neglecting the small contribution from the other two terms.

As a first step in computing the fluctuation term a 3D fluctuation structure has to be constructed that matches the scaling behaviour of the measured column density structure. This can be done in the following way. From the measured $\Delta$-variance spectrum of the density projection, i.e. the spectrum of the intensity map, we can compute the 3D $\Delta$-variance spectrum by de-projecting it according to the results from Sect. 2.5. By translating this spectrum by $k=z_{\text {tot }} / l$ into a spherically symmetric power spectrum $P(k)$, we create a new fBm-like structure using this power spectrum and random phases. This new structure should match the scaling behaviour of the input 2D $\Delta$-variance spectrum.

This is illustrated in Fig. 9, where we demonstrate the quality of this construction for two examples. In the first case we used the $\Delta$-variance spectrum of the projection of a known fBm with a spectral index $\beta=2.6$, while in the second we started from an artificial spectrum given by a power law corresponding to $\beta=3.7$

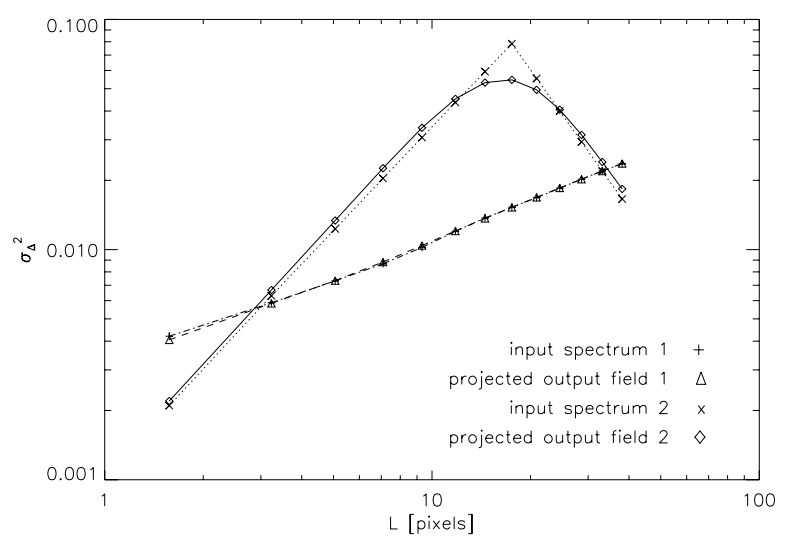

Fig. 9. Two examples for the construction of fluctuation fields from a projected $\Delta$-variance spectrum. The figure compares the input spectra with the spectra obtained from the $\Delta$-variance analysis of the projection of the computed fluctuation fields.

up to 17 pixels and an $l^{-2}$ decay above. In both cases we created the corresponding 3D fluctuation field, computed its 2D projection and the $\Delta$-variance spectrum of the projection. Comparing this derived spectrum with the input spectrum in Fig. 9 gives an impression of the quality of the reconstruction.

For the power-law input spectrum we find an almost perfect match with small deviations due to the artificial gridding of the fluctuation field, numerical uncertainties, and statistical fluctuations. In contrast, the spectrum composed of two powerlaws is less accurately reproduced. The $\Delta$-variance spectrum of the fluctuation field shows a broader peak and approaches the original spectrum only at lags relatively far apart from the peak. This broadening is due to the convolution of the power spectrum with the filter function in Eq. (7), which was ignored in the simple translation of the $\Delta$-variance spectrum back into the power spectrum described above. In principle, we could try to include a corresponding deconvolution to make the approach fully self-consistent, but the reasonable agreement between the two curves, even in this extreme case, shows that this additional refinement is not needed. The example was chosen to be extreme in the sense that we have a sharp turn from a steeply increasing spectrum into the steep decay of the $\Delta$-variance representing completely uncorrelated structures. In all cases with wider peaks, the agreement between the original spectrum and the derived spectrum is better, although the general tendency remains that the peak in the derived fluctuation spectrum is always slightly too broad. The actual quality of the construction of the fluctuation field from the $\Delta$-variance spectrum will thus fall between the two extremes shown in Fig. 9.

The fluctuation field constructed in this way has a zero average, so that we can use it directly as the auxiliary field to compute the centroids for $\rho-\rho_{0}$ in Fig. 8, i.e. the second term in Eq. (10). Unfortunately, the unknown field of velocity fluctuations also enters into this term, so that an iteration scheme is required; we start from the measured centroid spectrum, assuming that it is determined purely by the projection of the velocity field, then divide by $\rho_{0}^{2}$ and construct a fluctuation field for the velocities in the same way as described above for the density fluctuation field. From the convolution of the two fluctuation fields, we estimate the $\Delta$-variance spectrum of the correction term. Subtracting this spectrum from the measured centroid spectrum then provides the next estimate of the pure projection of the velocity field. This can be used again to determine the pure fluctuation term in the centroids and so on. The iteration 

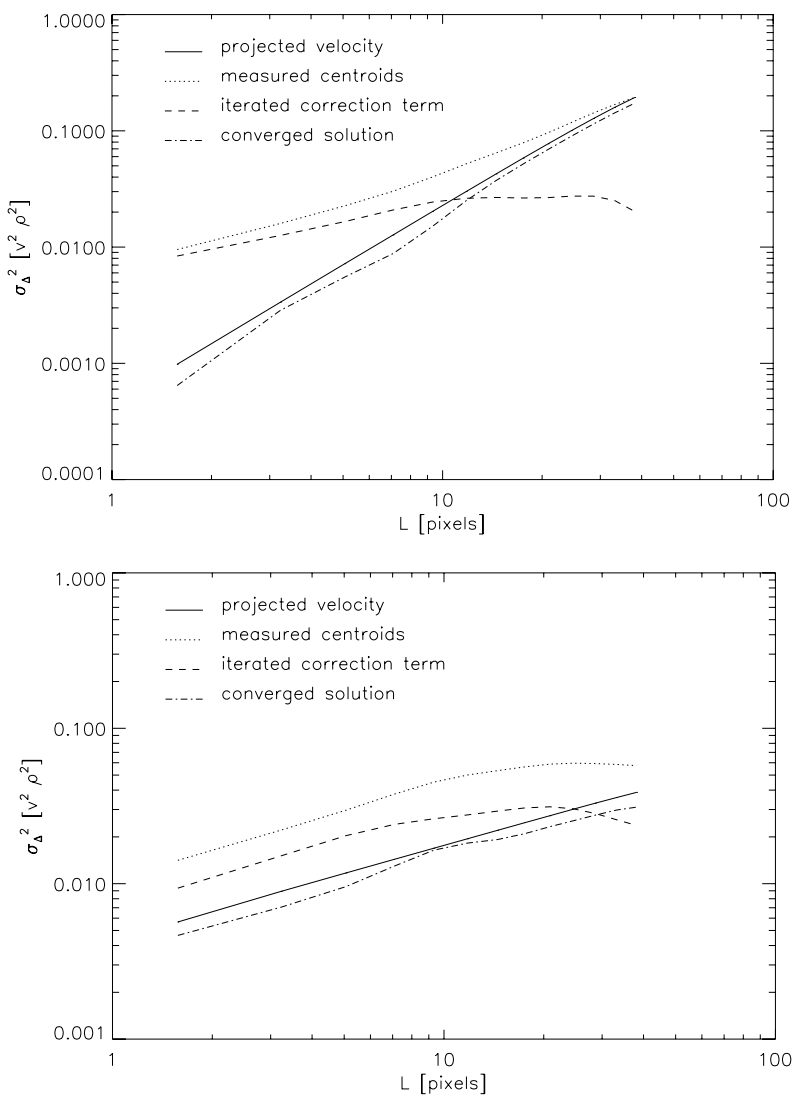

Fig. 10. $\Delta$-variance spectra of the weighted centroid velocities for the density and velocity structures from Fig. 8 (dotted lines). The solid line represents the projected velocity structure with a $\rho_{0}$ weighting. The dashed and the dash-dot lines represent the correction term and the derived projected velocity contribution at the end of the iteration. In the ideal case this converged solution should agree with the spectrum from the original velocity structure.

is stopped when the velocity spectrum obtained in subsequent steps remains constant within $1 \%^{7}$.

An example of the result of this iteration is displayed in Fig. 10 for the centroid spectra obtained from the combination of shallow density and steep velocity fields and vice versa, as shown in Fig. 8. The general recovery of the projected velocity structure is quite satisfactory. The absolute magnitude of the fluctuations is, however, somewhat too small in both cases, and the derived overall velocity spectrum is slightly steeper than the original spectrum for the combination of the shallow density with the steep velocity spectrum. These remaining deviations should stem from the accidental correlations between density and velocity field, expressed in the higher terms of Eq. (10). Altogether, the iteration scheme has proven to be a reliable method of recovering the original velocity spectrum from a measured centroid spectrum, when the projected density structure and the average density are known. In all $\mathrm{fBm}$ combinations tested here, the overall slope of the derived velocity spectrum agrees with that of the original spectrum within 0.1 . This is sufficient for distinguishing between different turbulence models (Elmegreen \& Scalo 2004).

7 The exact value of the convergence criterion is not important, because it only changes the number of required iterations. We found that the results obtained for smaller error limits cannot be distinguished by eye from the $1 \%$ limit results.

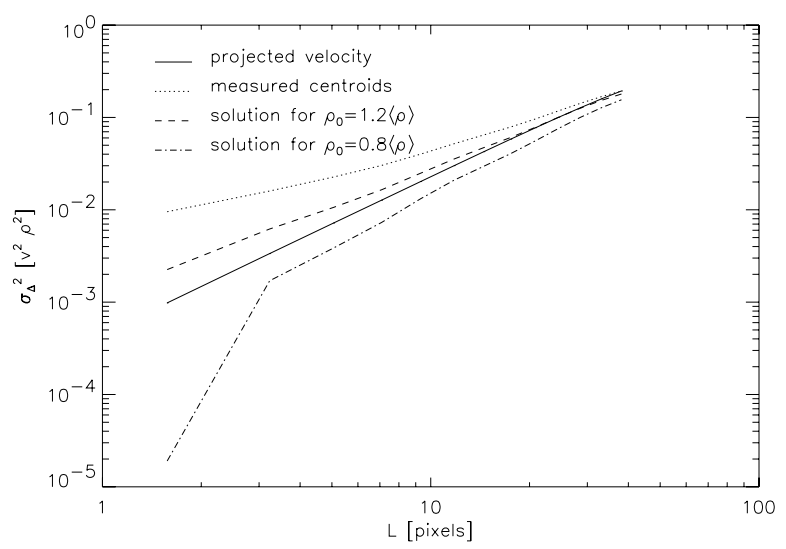

Fig. 11. Derivation of the projected velocity spectrum from a measured centroid spectrum when applying a $20 \%$ variation to the density $\rho_{0}$ used in the iteration scheme.

A major drawback of this method is the need to accurately estimate the average density in the considered interstellar cloud. This is not easy to obtain from the projected density in observational data because the line-of-sight extent of a cloud is often not known. This can be overcome in clouds with a known geometry or by excitation studies of molecular tracers sensitive to particular densities. However, very accurate estimates will always be difficult.

Thus we have studied the influence of an error in the average density on the reconstruction of the velocity structure. In Fig. 11 we have repeated the experiment shown in the upper plot of Fig. 10 when increasing and decreasing the average density relative to its actual value by $20 \%$. The result shows the same tendencies as discussed in Sect. 3.1. When the average density is overestimated, the centroids are thought to resemble the scaling of the underlying velocity structure better. The velocity fluctuations are underestimated because they are obtained by dividing the spectrum by a $\rho_{0}^{2}$ value that is too large. The computed correction term is too small and the derived velocity spectrum falls above the actual spectrum and is too shallow. If the average density is underestimated, we correct the centroid spectrum with an overestimated fluctuation spectrum, so that the derived velocity spectrum is too small and too steep. For steep velocity spectra a change in the average density by $20 \%$ corresponds to a change in the average exponent of the spectrum by 0.25 . For shallow spectra, the influence is somewhat smaller.

Thus we can conclude that it is possible to the retrieve the actual velocity spectrum from measured centroids even if the centroid spectrum is density-contaminated, but the accuracy of this retrieval depends critically on knowing the average density in the cloud. Independent measures of the $\sigma_{\rho} / \rho_{0}$ ratio are required. Methods for accurately deriving the density from multi-line observations have been successfully developed and applied e.g. by Schreyer et al. (1997); Richter et al. (2003); Sonnentrucker et al. (2003). They are based on a combination of information from different species tracing a wide range of critical densities, but the accurate determination of $\rho_{0}$ still remains a challenging task.

\section{Conclusions}

We have shown that the $\Delta$-variance analysis is an appropriate tool for characterising the scaling properties of both velocity centroid maps and the underlying 3D velocity field. By directly reflecting the power spectrum of fluctuations and preserving a power-law behaviour through the projection, the $\Delta$-variance 
is well suited to quantifying the properties of interstellar velocity fields. The fact that velocity centroids may not reflect the velocity statistics was always a concern for turbulence research. The disagreement between the aforementioned statistics was already discussed by Ossenkopf \& Mac Low (2002) and Brunt \& Mac Low (2004). We successfully tested the criterion for the validity of centroids as measures of velocity statistics as suggested by Lazarian \& Esquivel (2003).

We find that the most accurate criterion determining whether a centroid spectrum reflects the velocity scaling properties is a small ratio between the density dispersion and the mean density. The centroid spectra match the underlying velocity structure for values below 0.5 . Here, the centroids are determined by the pure projection of the velocity field. At higher $\sigma_{\rho} / \rho_{0}$ ratios, the mutual convolution of density and velocity fluctuation contributes a main term. Based on this knowledge, we can qualitatively explain all the differences in the interpretation of centroid spectra found in the literature.

Without knowing the average density in the considered medium, we can test whether a centroid spectrum reflects the true velocity structure using the criterion by Lazarian \& Esquivel (2003) that $X^{2} \sigma_{v_{\mathrm{c}}}^{2}(\boldsymbol{l}) \gg\left\langle v^{2}\right\rangle \sigma_{I_{\mathrm{int}}}^{2}(\boldsymbol{l})$ when the centroid spectrum is velocity-dominated. Although derived for the structure function, it holds for the $\Delta$-variance as well. However, there exists no unique factor by which the left hand side has to exceed the right hand side. We have confirmed the criterion by numerical experiments and found that factors above two are sufficient in the case of steep density spectra, but factors up to 100 may be required for extremely shallow density spectra. Currently, observations and simulations of interstellar turbulence show that both steep and shallow regimes may occur with density spectral indices ranging from about 2.5 to 3.3 (see Sect. 2.2).

We do not see any transition from velocity-dominated to density-dominated spectra at lower densities, but rather a transition to "density-contaminated" spectra that are systematically shallower. The flattening of the centroid spectra relative to the true velocity structure in the general case can be easily misinterpreted as a transition from spectra reflecting the velocity structure to spectra reflecting the density structure, because the density spectra are often shallower than the velocity spectra in interstellar turbulence. In any case, the density structure can be obtained directly from column density maps. A densitydominated spectrum occurs only if the velocity scale was chosen in an unfortunate way so that the average velocity is not negligible compared to the velocity dispersion. By adjusting the velocity frame such that the average line is centred at zero, this term can always be eliminated.

Whenever the centroid spectrum is velocity-dominated, the $\Delta$-variance analysis is a simple and robust tool for directly inferring the velocity scaling from the centroid map. The exponent of the $\Delta$-variance spectrum is the exponent of the power spectrum of the velocity fluctuations reduced by two. Although, the second-order structure function is connected to the power spectrum by a different functional behaviour, we find very similar behaviour when applied to centroid velocities. All general conclusions apply there as well. However, the structure functions of projections of power-law power spectra are always curved, so that a direct fit of the exponent is more difficult. Moreover, we find that, although our analytical decomposition of the velocity centroids is only valid for weighted centroids, the normalised centroids behave qualitatively in the same way so that they can also be used to derive the velocity structure whenever the centroid spectrum is velocity-dominated.
We provide an iteration scheme to derive the actual velocity structure from the centroid maps in all cases where the average density is known, even if the map is density-contaminated. An accurate determination of the power spectrum of the velocity fluctuations depends on three conditions: the correlation between density and velocity structure can be neglected, the dynamic range of length scales covered by the map is sufficient to compensate for statistical fluctuations at particular lags, and the average density $\rho_{0}$ can be estimated with high accuracy.

A different iteration scheme can be developed using the structure function instead of the $\Delta$-variance. In this case the decomposition proposed by Esquivel \& Lazarian (2005) can be used to obtain a scheme that is less sensitive to the knowledge of the average density, but a considerably more complex approach is needed to evaluate the projection effects. This will be the topic of a subsequent paper.

Acknowledgements. V.O. was supported by the Deutsche Forschungsgemeinschaft through grant 494A. A.E. acknowledges support from the NSF grant AST-0307869 and the Center for Magnetic Self-Organization in Laboratory and Astrophysical Plasmas, and Mexico's Consejo Nacional de Ciencia y Tecnología. A.L. is supported by NSF grant AST0307869. We thank an anonymous referee for comments helping to lay out many aspects in a clearer and more precise way. We have made use of NASA's Astrophysics Data System Abstract Service.

\section{References}

Bensch, F., Stutzki, J., \& Ossenkopf, V. 2001, A\&A, 366, 636

Brunt, C. M., \& Heyer, M. H. 2002, ApJ, 566, 289

Brunt, C. M., \& Mac Low, M.-M. 2004, ApJ, 604, 196

Chappell, D., \& Scalo, J. 1999, MNRAS, 310, 1

Cho, J., \& Lazarian, A. 2003, MNRAS, 345, 325

Cho, J., \& Lazarian, A. 2005, Theor. and Comput. Fluid Dynamics, 19, 127

Combes, F. 2000, in The Chaotic Universe, ed. V. G. Gurzadyan, \& R. Ruffini (World Sci.), 143

Deshpande, A. A., Dwarakanath, K. S., \& Goss, W. M. 2000, ApJ, 543, 227

Elemgreen, B. G., Kim, S., \& Staveley-Smith, L. 2001, ApJ, 548, 749

Elmegreen, B. G., \& Scalo, J. 2004, ARA\&A, 42, 211

Esquivel, A., \& Lazarian, A. 2005, ApJ, 631, 320

Esquivel, A., Lazarian, A., Pogosyan, D., \& Cho, J. 2003, MNRAS, 342, 325

Falgarone, E., Pineau Des Forêts, G., \& Roueff, E. 1995, A\&A, 300, 870

Falgarone, E., Panis, J.-F., Heithausen, A., et al. 1998, A\&A, 331, 669

Falgarone, E., Hily-Blant, P., \& Levrier, F. 2004, Ap\&SS, 292, 89

Goldreich, P., \& Shridhar, S. 1995, ApJ, 438, 763

Higdon, J. C. 1984, ApJ, 285, 109

Huber, D. 2002, Nonequilibrium, Self-Gravity and Fragmented Interstellar Medium, Ph.D.

Thesis No. 3348, Univ. Genève

Jenkins, E. 2004, Ap\&SS, 289, 215

Kleiner, S. C., \& Dickman, R. L. 1985, ApJ, 295, 466

Kim, J., \& Ryu, D. 2005, ApJ, 630, L45

Langer, W. D., Wilson, R. W., \& Anderson, C. H. 1993, ApJ, 408, L45

Lazarian, A. 1995, A\&A, 293, 507

Lazarian, A. 2004, JKAS, 37, 563

Lazarian, A., \& Esquivel, A. 2003, ApJ, 592, L37

Lazarian, A., \& Pogosyan, D. 2000, ApJ, 537, 720

Levrier, F. 2004, A\&A, 421, 387

Lis, D. C., Pety, J., Phillips, T. G., \& Falgarone, E. 1996, ApJ, 463, 623

Mac Low, M.-M., \& Ossenkopf, V. 2000, A\&A, 353, 339

Miesch, M. S., \& Bally, J. 1994, ApJ, 429, 645

Miesch, M. S., \& Scalo, J. M. 1995, ApJ, 450, L27

Miesch, M. S., Scalo, J., \& Bally, J. 1999, ApJ, 524, 895

Miville-Deschênes, M.-A., Levrier, F., \& Falgarone, E. 2003a, ApJ, 593, 831

Miville-Deschênes, M.-A., Joncas, G., Falgarone, E., \& Boulanger, F. 2003b, A\&A, 411, 109

Münch, G. 1958, Rev. Mod. Phys., 30, 1035

Ossenkopf, V. 2002, A\&A, 391, 295

Ossenkopf, V., \& Mac Low, M.-M. 2002, A\&A, 390, 307

Ossenkopf, V., Bensch, F., \& Stutzki, J. 2000, in The Chaotic Universe, ed. V. G. Gurzadyan, \& R. Ruffini, World Sci., 394

Ossenkopf, V., Krips, M., \& Stutzki, J. 2005, A\&A, submitted

Padoan, P., Jones, J. T., \& Nordlund, A. P. 1997, ApJ, 474, 730

Padoan, P., Boldyrev, S., Langer, W., \& Nordlund, A. P. 2003, ApJ, 583, 308

Peitgen, H.-O., \& Saupe, D. 1988, The Science of Fractal Images (Berlin: Springer)

Richter, P., Sembach, K. R., \& Howk, J. C. 2003, A\&A, 405, 1013

Schreyer, K., Helmich, F. P., van Dishoeck, E. F., \& Henning, T. 1997, A\&A, 326, 347

Sonnentrucker, P., Friedman, S. D., Welty, D. E., York, D. G., \& Snow, T. P. 2003, ApJ, 596, 350

Stanimirović, S., \& Lazarian, A. 2001, ApJ, 551, L53

Stenholm, L. G. 1984, A\&A, 137, 133

Stutzki, J., Bensch, F., Heithausen, A., Ossenkopf, V., \& Zielinsky, M. 1998, A\&A, 336, 697 Zank, G. P., \& Matthaeus, W. H. 1992, J. Plasma Phys., 48, 85 